\title{
Loschmidt's Paradox, Extended to CPT Symmetry, Bypasses Second Law
}

\author{
George S. Levy \\ Entropic Power, Irvine, California, USA \\ Email: glevy@entropicpower.com
}

How to cite this paper: Levy, G.S. (2019) Loschmidt's Paradox, Extended to CPT Symmetry, Bypasses Second Law. Journal of Applied Mathematics and Physics, 7, 3140-3175.

https://doi.org/10.4236/jamp.2019.712221

Received: November 22, 2019

Accepted: December 17, 2019

Published: December 20, 2019

Copyright $\odot 2019$ by author(s) and Scientific Research Publishing Inc. This work is licensed under the Creative Commons Attribution International License (CC BY 4.0).

http://creativecommons.org/licenses/by/4.0/

\begin{abstract}
Loschmidt's paradox is extended by replacing its assumption of time reversibility with full CPT symmetry. Mobility is identified as a means for expressing collisions or dissipation, and the cross product of its gradient with the magnetic field, for expressing parity. Three phenomena incorporating such cross products are identified. The first is the cross product of the mobility gradient with the magnetic field. The second combines this cross product with the E cross B drift. The third is the reciprocal of the Nernst effect expressed as a cross product of the temperature gradient and the magnetic field. Simulations are conducted for testing Loschmidt's extended paradox. Onsager's exclusion of magnetic fields and rotation from reciprocals violates CPT symmetry and is unjustified. All three cross-product phenomena skew statistics in a fashion unanticipated by Boltzmann's assumptions in his H-Theorem. CPT symmetric systems fall outside the assumptions of the theorem which is not rendered invalid but simply limited to its domain of applicability. Therefore, these systems do not violate the second law as Boltzmann defines it. They bypass it.
\end{abstract}

\section{Keywords}

Second Law, Boltzmann, Loschmidt, CPT Symmetry, Entropy, Nernst Effect, H-Theorem, Onsager, Paradox, Cross Product

It is the only physical theory of universal content concerning which I am convinced that, within the framework of the applicability of its basic concepts, it will never be overthrown-Albert Einstein on the Second Law [1].

\section{Introduction}

The second law, as stated in Boltzmann's H-Theorem [2] asserts that, on the av- 
erage, entropy in an isolated system never decreases with time. i.e., $\mathrm{d} S / \mathrm{d} t \geq 0$. Joseph Loschmidt [3] [4] objected to the proof. In his famous paradox, he argued that if all physical processes are truly microscopically time-reversible, then any entropy increasing process is as probable as a corresponding entropy decreasing process. Therefore, according to physical laws, the change in entropy must be zero. Boltzmann's response was to assert that molecular chaos (Stosszahlansatz), i.e. uncorrelated molecular collisions, increases the randomness of a system. His molecular chaos assumption, however, implicitly assumes the arrow of time [5]. A time-reversed molecular chaos would lead to an anti-second law result, i.e., $t \rightarrow-t$ leads to $\mathrm{d} S /(-\mathrm{d} t) \leq 0$. Boltzmann's explanation, therefore, is no explanation at all, but simply the refutation of Loschmidt's time reversibility premise: the arrow of time is a fundamental assumption implicitly used by Boltzmann in the $\mathrm{H}$-Theorem which is his proof of the second law.

Other proofs by Gibbs [6] [7], Tolman [8], and Von Neuman [9] [10] also implicitly rely on the arrow of time. One of the most recent, the fluctuation theorem [11] [12] [13] shows that the probability of observing an occurrence of entropy production opposite to the second law decreases with the time of observation. However, as Evans and Searles [12] [13] point out, this theorem must be combined with the axiom of causality. Their solution implicitly assumes the arrow of time and, regardless, is not workable as it violates locality (see Appendix A).

In view of advances in the physics of the twentieth and twenty-first century, Loschmidt's paradox appears to be based on a narrowly focused premise. Laws of nature, as currently understood, are not simply time-reversible. Their symmetry includes charge, parity and time reversal in combination. Therefore, simply asserting the arrow of time is not a sufficient condition for validating or falsifying the second law.

As Carl Popper famously remarked, physical laws can never be shown to be true, but they should be experimentally falsifiable. On the other hand, mathematical theorems can be proven from a set of axioms. The H-Theorem falls into this second category as it is derived from assumptions and postulates such as the Fundamental Postulate of Thermodynamics. As of now, all such proofs of the second law supervene on the assumption of the arrow of time.

This paper challenges the second law by extending Loschmidt's paradox as follows: if the laws of nature are CPT symmetrical and reversible, then a system could return to a previous state even in the presence of an arrow of time, thereby restoring its entropy to its original value. This version of the paradox renders moot the arrow of time assumption. This paper does not resolve the mystery of the arrow of time. However, it shows that systems with CPT symmetry skew particles' statistics in a fashion unanticipated by Boltzmann, making them fall outside assumptions of his H-Theorem. Referring to Einstein's quote at the beginning of this paper, on the second law, CPT symmetric systems do not fit within the framework of the applicability of its basic concepts. Therefore, the second law, as Boltzmann defines it, is not violated, it is bypassed. 
The idea of applying CPT symmetry to thermodynamics is not new. Klimenko [14] [15] and Klimenko and Maas [16] show that the kinetics of antimatter systems is time-antisymmetric when CPT invariance is taken into consideration. In contrast, this paper presents a classical analysis that mainly covers conventional matter systems in which the charge is constant, that is, these systems comply with PT symmetry. However, the results are applicable to CPT symmetry.

Physical phenomena capable of expressing parity in CPT symmetry require a cross product, typically with a magnetic field (i.e., $\times B$ ). Interestingly, Onsager [17] [18] excluded magnetic fields from his reciprocals because of a conflict with the second law. This paper shows that this exclusion is not justified (discussed in Appendix B). Turned on its head, his argument can be taken as a proof that systems that include magnetic fields or rotations can violate the second law.

A phenomenon that contests the second law must also include a measure of particle collisions (Boltzmann's molecular chaos or Stosszahlansatz) tightly woven into the selected CPT symmetry cross-product mechanism. Mobility is such a measure. This tight coupling can be achieved by using the mobility gradient $\nabla \mu$ as the other factor in the aforesaid cross product.

Therefore, a system that extends Loschmidt's paradox must combines CPT symmetry with Boltzmann's molecular chaos. This system must express charge, parity, time, and mobility. It could be a gas of charged particles (Charge) whose behavior (Time) is described by a cross product (Parity) between a mobility gradient $\nabla \boldsymbol{\mu}$ (collisions) and a magnetic field $\boldsymbol{B}$.

Three such cross-product phenomena have been identified and simulations have been conducted as means for challenging the second law. This paper is organized as follows:

1) Section 2 covers the $\nabla \boldsymbol{\mu} \times \boldsymbol{B}$ drift. This phenomenon gives rise to spontaneous circulation driven by ambient thermal energy.

2) Section 3 covers the $\nabla \boldsymbol{\mu} \times \boldsymbol{B}+\boldsymbol{E} \times \boldsymbol{B}$ drift which produces spontaneous space charges and voltages.

3) Section 4 covers the reciprocal of the Nernst Effect $(\nabla T \times B)$ which generates spontaneous temperature gradients.

4) Section 5 describes simulation experiments supporting the theory.

5) Section 6 explains that the thesis advanced in this paper does not break the second law as proven by Boltzmann and others, it bypasses it.

6) Section 7 provides experimental supporting evidence.

7) Section 8 is the conclusion.

8) Section 9 suggests future research projects.

\section{The $\nabla \mu \times B$ Drift}

Charged particles in a medium configured with a mobility gradient and subjected to a magnetic field perpendicular to the gradient, drift in a direction perpendicular to the gradient and the field. This effect can, in general, be described as a cross product: 


$$
\boldsymbol{v}_{\mu \times B} \propto \nabla \boldsymbol{\mu} \times \boldsymbol{B} .
$$

A mobility gradient can give rise to a force. See Note in [19]. This effect, first introduced as a surface drift in a previous paper by this author [20] [21], shall be elaborated upon below.

The simplest configuration enabling the $\nabla \boldsymbol{\mu} \times \boldsymbol{B}$ drift is shown in Figure 1 (a). It comprises a surface in the $\mathrm{XZ}$ plane, separating a medium with zero mobility, except absorption and emission (for example the floor of a vacuum chamber) from a medium with infinite mobility (the volume of the chamber). Other surface effects such as image potentials are ignored for simplicity. In this simplified case, the mobility function is a step from zero to infinity at the surface, producing an impulse gradient in a direction normal to the surface i.e., along the $\mathrm{Y}$ axis. When a magnetic field $B$ is applied parallel to the surface along the $\mathrm{Z}$ axis, a particle starting out from the surface follows a circular orbit that eventually brings it back to the surface. The surface interaction generates a drift along the $\mathrm{X}$ axis through two mechanisms:

1) Position Distribution Skewing.

2) Velocity Distribution Skewing.

\subsection{Position Distribution Skewing}

Figure 1(a) shows a charged particle following an orbit under the influence of a magnetic field $\boldsymbol{B}$ in a quasi-vacuum chamber, as it is being emitted and absorbed by a surface.

If the orbit is clockwise (i.e., $q B_{z}>0$ ) and the surface is the floor (zero mobility medium below the surface), then the end point is always to the right of the starting point, and vice versa, if the surface is the ceiling (zero mobility medium above the surface), the end point is always to the left. Remarkably, the only conditions for statistical biasing are 1) charged particles, 2) a magnetic field or rotation and 3) the nominal distribution, undisturbed by a magnetic field, is symmetric and unbiased. Otherwise, positional biasing occurs independently of the properties of the surface which could be a perfect reflector, emitter, absorber or thermalizer. Figure 1 (b) shows a simulation of all the paths a particle could take for its range of velocity vectors defined by the Maxwell-Boltzmann distribution. Green represents the peak of the distribution, blue the most energetic particles and red, the least energetic ones.

\subsection{Velocity Distribution Skewing}

A gas at any arbitrarily close distance to the surface has a symmetrical velocity distribution as shown in Figure 2(a) for the following reason.

A perfectly reflecting or thermalizing surface behaves as an imaginary partition separating an imaginary volume also containing gas. Any particle emitted or absorbed by the surface can be regarded as crossing this partition and leaving or entering this imaginary volume. Therefore, the velocity distribution of all the particles at any given point arbitrarily close to the partition, is perfectly 


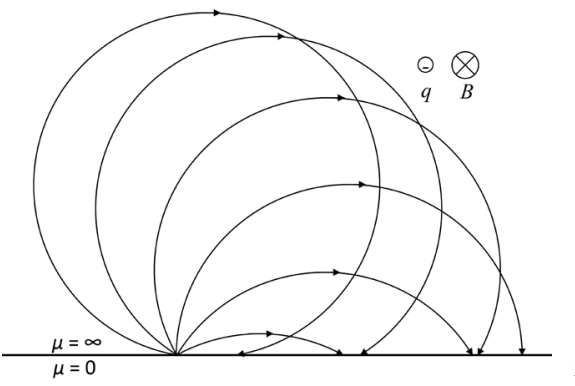

(a)

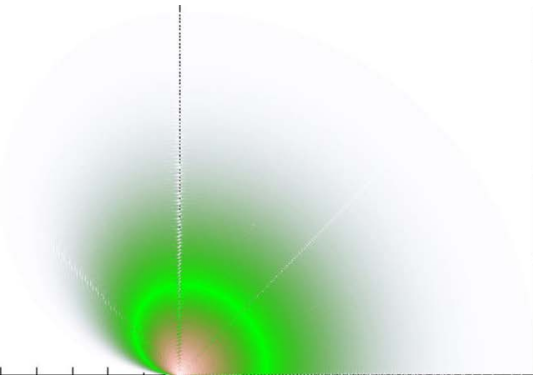

(b)

Figure 1. (a) When a magnetic field is present, a charged particle starting out from the floor follows a circular orbit that always intersects the surface to the right when the orbit is clockwise and to the left when it is counterclockwise, (b) This image shows a simulation of a large number of orbits with a maxwellian velocity distribution. Blue indicates orbits with the most energy, green those at the peak of the distribution, and red those with the least energy. Particles are assumed to have a negative charge and the magnetic field is "into the paper".

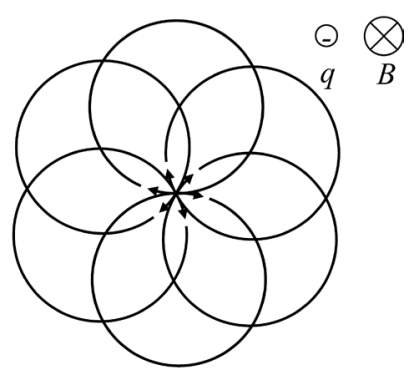

(a)

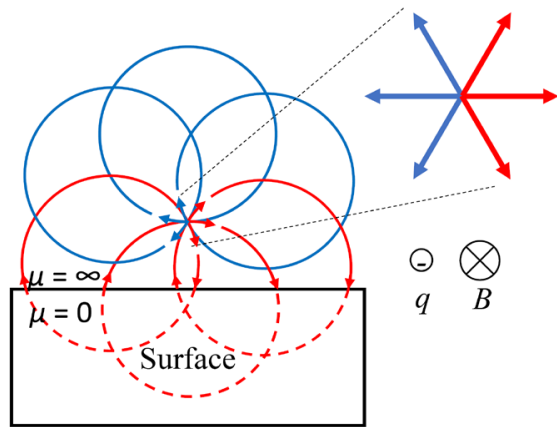

(b)

Figure 2. (a) The velocity distribution of particles at any given point is unbiased; (b) However, when a surface is present, those particles (in red) that do make contact with the surface have a velocity distribution skewed in one direction, and those (in blue) that do not make contact have a distribution in the opposite direction. The asymmetry results in a transfer of momentum between the gas and the surface. In the figure, particles are assumed to have a negative charge and the magnetic field is "into the paper".

symmetrical and has zero bias-as if the surface did not exist. At the surface itself, the distribution can be considered "half-Maxwellian" but it is still symmetrical around the normal to the surface, as if the surface did not exist. (This model is consistent with Kirchhoff's law of thermal radiation).

However, the surface still introduces a subtle asymmetry as shown in Figure 2(b). The velocity distribution of the subset of particles at a given point, with trajectories intersecting the surface (cross the partition) is biased as shown in red. Conversely, the subset of particles not making contact is biased in the other direction as shown in blue. The combined distribution has zero bias. Yet the surface introduces a correlation which transfers momentum between the gas and the surface.

Figure 3(a) and Figure 3(b) show the skewing of the Maxwell Boltzmann distribution near a surface. 


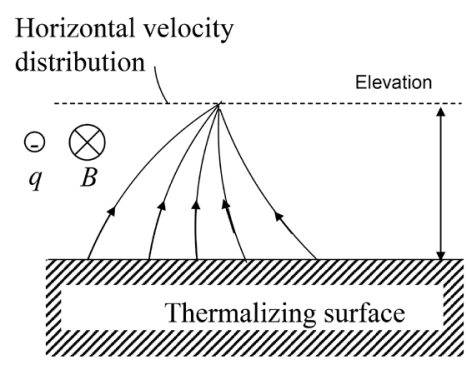

(a)

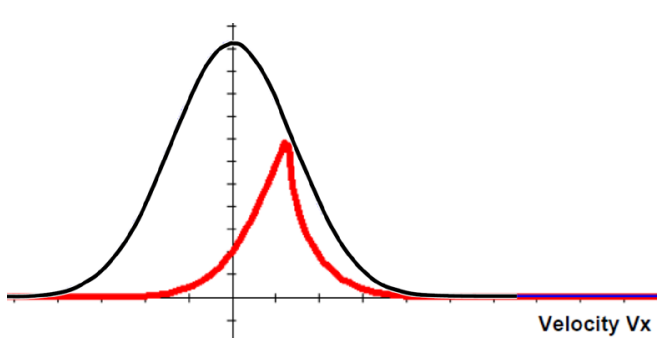

(b)

Figure 3. (a) In a magnetic field, particles have a distribution biased in one direction when they intersect a surface, and in the opposite direction when they don't; (b) The black curve represents the unbiased normalized Maxwell-Boltzmann velocity distribution for all particles corresponding to Figure 2(a). The red curve corresponding to Figure 2(b) is the distribution of the subset of particles at an elevation of $300 \mathrm{~nm}$, having a trajectory that intersects the surface, for a magnetic field of 0.2 Tesla. Velocity is displayed along the $\mathrm{X}$ axis with $4039 \mathrm{~m} / \mathrm{s}$ per division.

\subsection{Circulation Due to Momentum Transfer in the $\nabla \mu \times B$ Drift}

The $\nabla \boldsymbol{\mu} \times \boldsymbol{B}$ drift produces circulation of the gas within the container, in a direction opposite to the orbits for the following reason. When an orbit is truncated by a surface, momentum is transferred from the surface to the rest of the gas in a direction opposite to the orbital rotation. As shown in Figure 4(a), clockwise orbits produce a counterclockwise circulation and vice versa, in accordance with Newton's third law. It is the macroscopic reaction to the commonly accepted microscopic cyclotron orbits. Figure 4(b) shows a simulation of this phenomenon. This circulation is spontaneous, driven by thermal energy transferred to the gas thermalized by the walls, floor and ceiling, with no input current.

It may be experimentally verified in a modified Crookes radiometer [22] in thermal equilibrium with a heat bath and in a vertical magnetic field. In contrast with conventional devices, this radiometer includes vanes with equally colored faces and an opaque bulb and is driven by the thermal energy of the gas. A more detailed description of this experiment is presented in section 9 Suggested Future Research. This experiment is a magnetic version of Duncan's paradox [23].

\subsection{Analysis of the $\nabla \mu \times B$ Drift}

Consider particles with charge $q$ and an effective mass $m^{*}$ subjected to a magnetic field $B_{z}$ applied along the $\mathrm{Z}$ axis. The particles are emitted by a surface in the $\mathrm{XZ}$ plane into a medium above it, filling the XYZ volume. The surface is assumed to have zero mobility except for the ability to emit and absorb particles. Surface effects such as image potentials are ignored. The medium is assumed to have mobility $\mu$. Two types of media shall be discussed with the following simplifying assumptions:

1) Medium with infinite mobility.

2) Medium with a small but finite mobility. 


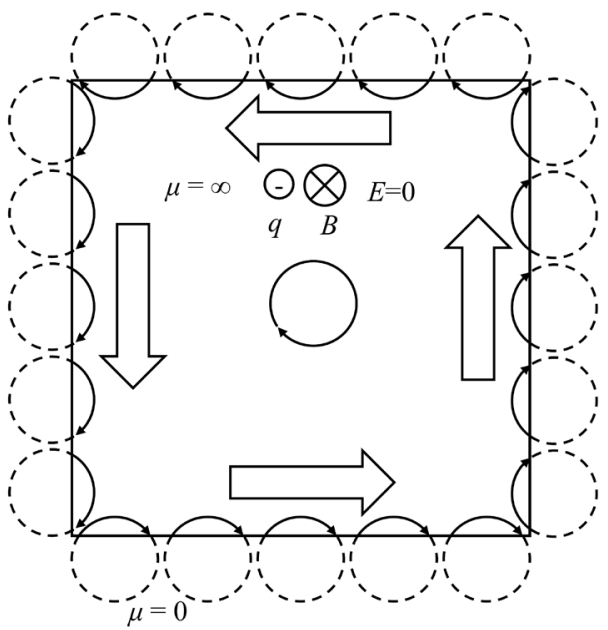

(a)

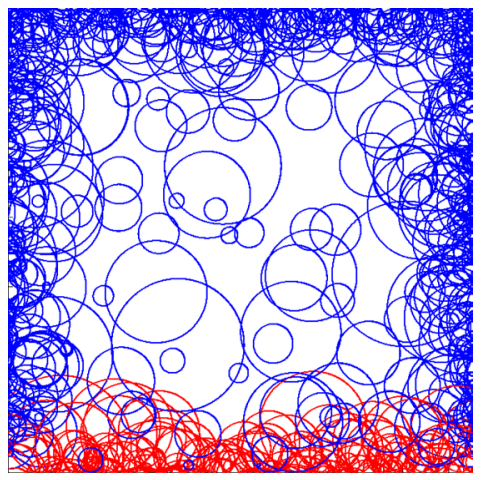

(b)

Figure 4. (a) The truncated orbits drive circulation of charged particles around the chamber; (b) A simulation shows in red those trajectories moving to the right, and in blue, those moving left, up and down. The greater concentration of particles around the edges is due to their mutual repulsion (enhanced by the simulation).

\subsubsection{Case 1. Medium with Infinite Mobility}

This case is illustrated in Figure 5. Mobility in the medium is assumed to be infinite, such that a particle leaving the surface with a tangential angle $\theta_{0}$ eventually collides with the surface after following an uninterrupted arc of radius $R$, subtending angle $\Delta \theta$. The time taken by the particle travelling at velocity $v_{x y}$ projected on the XY plane, to cross the $\operatorname{arc} R \Delta \theta$ is the same as the time taken by the particle traveling at drift velocity $v_{\mu \times B}$ projected on the surface, to cross the chord $2 R \sin (\Delta \theta / 2)$.

$$
\text { Travel Time }=\frac{R \Delta \theta}{v_{x y}}=\frac{2 R \sin (\Delta \theta / 2)}{v_{\mu \times B}} .
$$

Since $\Delta \theta=2 \theta_{0}$,

$$
v_{\mu \times B}=v_{x y} \frac{\sin \left(\theta_{0}\right)}{\theta_{0}} .
$$

The expectation value of $v_{x y}$ and $v_{\mu \times B}$ are derived in Appendix $C$ for the Maxwell-Boltzmann distribution. They are

$$
\bar{v}_{x y}=\sqrt{\frac{\pi k_{B} T}{2 m^{*}}},
$$

and

$$
\bar{v}_{\mu \times B}=\operatorname{Si}(\pi) \bar{v}_{x y}=\operatorname{Si}(\pi) \sqrt{\frac{\pi k_{B} T}{2 m^{*}}} .
$$

The sign of $\bar{v}_{\mu \times B}$ depends on the sign of $q B_{z}$ and the orientation of the surface. For example, if the gas particles are contained by a cubic chamber with edges along the $\mathrm{X}, \mathrm{Y}$ and $\mathrm{Z}$ axes (as shown in Figure 4(a), when $q B_{z}>0$, the surface drift is to the right on the floor, upward on the right wall, to the left on 


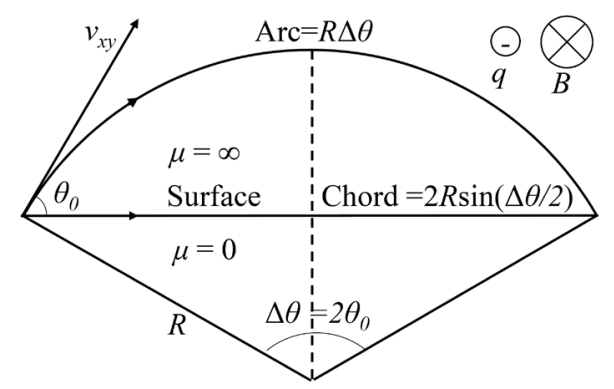

Figure 5. Drift velocity diagram. A particle leaves the surface, and follows an uninterrupted arc that brings it back to the surface.

the ceiling and downward on the left wall. This results in a spontaneous circulation around the chamber. Figure $4(\mathrm{~b})$ is a simulation showing in red the trajectories of particles moving to the right, and in blue, those moving up, down and to the left.

\subsubsection{Case2. Medium with Small But Finite Mobility}

For a given mobility $\mu$, the mean free time $\tau$ can be expressed as $\tau=\mu m^{*} / q$. For a velocity $v$, the mean free path is:

$$
\lambda=\frac{\mu m^{*} v}{q} .
$$

Therefore, the projection of the mean free path $\lambda_{x y}$ and velocity $v_{x y}$ on the $\mathrm{XY}$ plane is

$$
\lambda_{x y}=\frac{\mu m^{*} v_{x y}}{q} .
$$

Referring to Figure 6, the $\nabla \boldsymbol{\mu} \times \boldsymbol{B}$ drift can be derived as follows. The length of the arcuate mean free path projection on the XY plane between collision1 at the surface and collision 2 in the medium is:

$$
\lambda_{x y}=R \Delta \theta,
$$

where $\Delta \theta$ is the angle subtended by the arcuate path between the initial and final velocity vector and $R$ is the radius of curvature. The angle $\Delta \theta$, or equivalently the $\mu B_{z}$ product (see Equation (13)) shall be assumed to be small to allow simplifying trigonometric approximations. Half the drift between the linear path and the arcuate path is:

$$
R \Delta \theta^{2} / 2 \text {. }
$$

The full drift distance projected on the $\mathrm{X}$ axis is:

$$
R \Delta \theta^{2} \sin \left(\theta_{0}\right) \text {. }
$$

The time taken by the particle travelling at $v_{x y}$ to cross the arc $\lambda_{x y}=R \Delta \theta$ (Equation (8)) is the same as the time taken by the particle traveling at drift velocity $v_{\mu \times B}$ to cross the full drift distance along the surface (Equation (10)). Hence:

$$
\text { Traveling time }=\frac{R \Delta \theta}{v_{x y}}=\frac{R \Delta \theta^{2} \sin \left(\theta_{0}\right)}{v_{\mu \times B}} .
$$




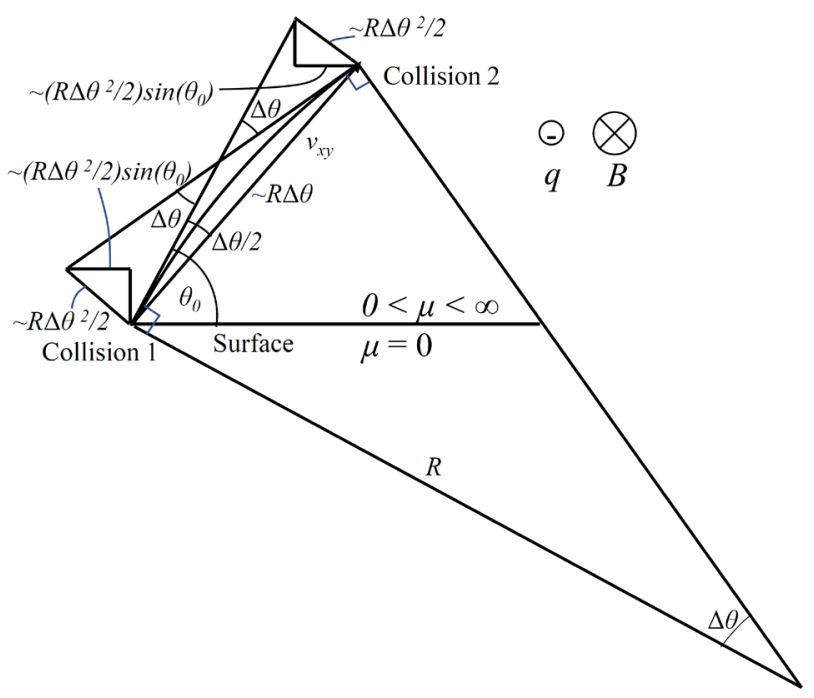

Figure 6. In a finite mobility medium, a particle leaves the surface, travels an arc of length $\lambda_{x y}=R \Delta \theta$ until it experiences a collision. The drift along the $\mathrm{X}$ axis due to the magnetic field is $R \Delta \theta^{2} \sin \left(\theta_{0}\right)$. The trigonometric results are only valid for small values of $\Delta \theta$.

Therefore,

$$
v_{\mu \times B}=v_{x y} \Delta \theta \sin \left(\theta_{0}\right) .
$$

Combining Equations (6) and (8), and since $R=m^{*} v_{x y} / q B_{z}$ one gets:

$$
\frac{\lambda_{x y}}{R}=\Delta \theta=\mu B_{z} .
$$

Hence the drift velocity as a function of $\theta_{0}$ is:

$$
v_{\mu \times B}=v_{x y} \mu B_{z} \sin \left(\theta_{0}\right)
$$

Since $v_{x y}, \mu$, and $B_{z}$ are independent of $\theta_{0}$, and $\int \sin \left(\theta_{0}\right) \mathrm{d} \theta_{0}=2$ between 0 and $\pi$, one can integrate Equation (14) for $0<\theta_{0}<\pi$, to obtain the expected drift velocity $\bar{v}_{\mu \times B}$, yielding:

$$
\bar{v}_{\mu \times B}=2 \bar{v}_{x y} \mu B_{z} .
$$

The expected velocity $\bar{v}_{x y}$ is derived in Appendix C in Equation (75). Combining with Equation (15) the expected drift velocity $\bar{v}_{\mu \times B}$ can be expressed as:

$$
\bar{v}_{\mu \times B}=\sqrt{\frac{2 \pi k_{B} T}{m^{*}}} \mu B_{z} .
$$

As already mentioned in relation to Figure 4(a), the sign of $\bar{v}_{\mu \times B}$ depends on the sign of $q B_{z}$ and the orientation of the surface. When $q B_{z}>0$, the surface drift is to the right on the floor, upward on the right wall, to the left on the ceiling and downward on the left wall.

The term $\mu$ in Equation (16) represents the mobility of the medium over a surface with mobility of zero. The mobility function is a step in a direction normal to the surface, i.e., along the $\mathrm{Y}$ axis. Furthermore, $\bar{v}_{\mu \times B}$ occurs along the $\mathrm{X}$ axis. Therefore, this equation can be rewritten in a more generalized vector form as: 


$$
\overline{\boldsymbol{v}}_{\mu \times B}=\sqrt{\frac{2 \pi k_{B} T}{m^{*}}} \boldsymbol{\mu} \times \boldsymbol{B} .
$$

Since $\boldsymbol{\mu}$ is a step function, it can be taken as a differential step along a perpendicular to the surface. Expressed more generally, mobility can be configured with a gradient $\nabla \boldsymbol{\mu}$ normal to the surface. Equation (17) can then be rewritten as:

$$
\nabla \overline{\boldsymbol{v}}_{\mu \times B}=\sqrt{\frac{2 \pi k_{B} T}{m^{*}}} \nabla \boldsymbol{\mu} \times \boldsymbol{B},
$$

where the velocity gradient is parallel to the mobility gradient. The $\nabla \boldsymbol{\mu} \times \boldsymbol{B}$ drift can be viewed as generated by an entropically generated force (see Appen$\operatorname{dix} \mathrm{D})$.

Systems discussed in this section have a mean free path comparable to the radius of the particles' orbits, implying that they operate below the thermodynamic limit [24] which, as Sheehan points out [25], leads to unusual effects. Assuming a surface with a step function in mobility, then one could use Equation (13) to define an index $\eta$ as a measure of how close one is to this limit:

$$
\eta=\frac{\lambda_{x y}}{R}=\Delta \theta=\mu B_{z} .
$$

(In general, $\mu$ can be continuous rather than a step function. Therefore, a more general definition would be $\eta=\Delta \mu B_{z}$ ). The thermodynamic limit is reached when the mean free path XY projection $\lambda_{x y}$ is very small in comparison with the orbital radius, i.e., $\lambda_{x y} \ll R$ or equivalently, when $\eta$ or $\mu B_{z}$ tend to zero. At that point, the behavior of particles become symmetrical in space as if the magnetic field did not exist, and the surface drift velocity $\bar{v}_{\mu \times B}$ as defined by Equations (16), (17) and (18) goes to zero. In contrast, when the mean free path is large, a strong surface drift occurs. For example, when $\eta=2 \pi$ then $\lambda_{x y}$ is equal to the circumference of an orbit. This result highlights two important parameters in the design of a device using the $\boldsymbol{E} \times \boldsymbol{B}$ drift:

1) The carrier mobility should be high. Among semiconductors, Indium Antimonide (InSb) has an electron mobility $\mu=7.7 \mathrm{~m}^{2} \cdot \mathrm{V}^{-1} \cdot \mathrm{s}^{-1}$, at $T=300 \mathrm{~K}$, one of the highest known [26].

2) The magnetic source should be capable of generating a strong magnetic flux density. A neodymium or iron nitride magnet can produce a field of at least 1 Tesla.

In a device employing InSb with $B=1$ Tesla and $T=300 \mathrm{~K}$, the thermodynamic limit index is $\eta=7.7$. This implies that $\lambda_{x y}$ is 1.226 times longer than the circumference of an orbit of radius $R$, sufficiently high to support a usable $E \times B$ drift.

\section{The $\nabla \mu \times B+E \times B$ Drift}

This section shows that, in the presence of a magnetic field, a mobility gradient in tandem with an electric field can cause particles to accumulate to produce 
space charges and voltages. The following shall be discussed:

1) The $E \times B$ drift shall be briefly reviewed.

2) The $\nabla \boldsymbol{\mu} \times \boldsymbol{B}$ and $\boldsymbol{E} \times \boldsymbol{B}$ in combination.

\subsection{The $E \times B$ Drift}

In general, when a force $F$ acts on particles with charge $q$, and in the presence of a magnetic field $B$, the particle drift with an average velocity $\boldsymbol{v}_{E \times B}$ in a direction defined by the cross product of $\boldsymbol{F}$ and $\boldsymbol{B}$, which can be expressed as [27]

$$
\boldsymbol{v}_{E \times B}=\frac{1}{q} \frac{\boldsymbol{F} \times \boldsymbol{B}}{\boldsymbol{B}^{2}} .
$$

More specifically, particles with charge $q$ subjected to an electric field $E$ experience a force $F=q E$. Therefore, according to Equation (20) the $\boldsymbol{E} \times \boldsymbol{B}$ drift can be expressed as

$$
\boldsymbol{v}_{E \times B}=\frac{\boldsymbol{E} \times \boldsymbol{B}}{\boldsymbol{B}^{2}} .
$$

When $\boldsymbol{B}$ and $\boldsymbol{E}$ are perpendicular to each other, the vector Equation (21) can be simplified to:

$$
v_{E \times B}=\frac{E_{y}}{B_{z}},
$$

where $\boldsymbol{v}_{E \times B}$ is in the $\mathrm{X}$ direction. The $\boldsymbol{E} \times \boldsymbol{B}$ current along the $\mathrm{X}$ axis can then be expressed as

$$
J_{E \times B}=n q \frac{E_{y}}{B_{z}} .
$$

A detailed analysis of the $E \times B$ drift is provided in Appendix E. Equation (23) holds only when the mobility is infinite. In media with finite mobility the magnitude of the drift current is given by Equation (96) in Appendix E:

$$
|\boldsymbol{J}|=\frac{n q \mu B_{z}}{\sqrt{1+\left(\mu B_{z}\right)^{2}}} \frac{E_{y}}{B_{z}},
$$

and the direction of the drift is determined by the mobility of the particles and shifted away from the $\mathrm{X}$ axis according to Equation (97) in Appendix E:

$$
\theta_{E \times B}=\tan ^{-1}\left(\frac{1}{\mu B_{z}}\right) \text {. }
$$

The thermodynamic limit index $\eta=\mu B_{z}$ determines the direction of the drift and its magnitude.

\subsection{The $\nabla \mu \times B$ and $E \times B$ Drifts in Combination}

Upon close examination, the $E \times B$ drift cannot be separated from surface effects. When the mobility is uniform throughout the medium and for perpendicular fields $E_{y}$ and $B_{z}$, the drift occurs in a straight line at an angle $\theta_{E \times B}$ from $\mathrm{X}$ axis. When $\mu=\infty$, the drift is along the $\mathrm{X}$ axis $\left(\theta_{E \times B}=0\right)$ as per Equation (97). When 
$\mu$ is finite, $\theta_{E \times B}$ is defined by Equation (21). Nevertheless, in any physical implementation, the drift must eventually encounter a boundary such as the wall of a plasma chamber or the surface of a high performing (high mobility) semiconductor.

The charged particles then accumulate on this boundary thereby producing local variations in particle density, (i.e., space charges). These voltages have been observed in plasma experiments. [28] [29] [30] and in simulation.

The Hall Effect (i.e., the reciprocal of the $E \times B$ drift) also requires surfaces onto which particles can accumulate and across which a measurable voltage can be measured. No Hall Effect voltage could possibly be measured in a particle container of infinite size (or has wrap around walls as can be done in simulations).

The role of surfaces in these effects is often implicitly accepted, yet it is fundamental. Since a surface is characterized by an abrupt change in mobility, one can generalize the concept of surface by replacing it with mobility gradient. Accordingly, the $E \times B$ drift cannot be considered independently of mobility gradient in general and of surfaces in particular, and of their thermalizing influence of mobility on particles. Therefore, the $E \times B$ drift must be analyzed in conjunction with the $\nabla \boldsymbol{\mu} \times \boldsymbol{B}$ drift of Section 2. Figure 7 shows both kinds of drifts in a chamber. The drifts point in the same direction at the ceiling but in opposite directions at the floor.

As shown in Figure 7 and Figure 8(a), electrical carriers in the bulk are carried by the $E \times B$ drift until they collide with a surface (floor, ceiling or wall.) where both drifts operate sometimes additively, sometime subtractively. While the direction of the $E \times B$ drift is only dependent on the cross product of $E$ and $\boldsymbol{B}$ and independent of the charge of the particles, the direction of the

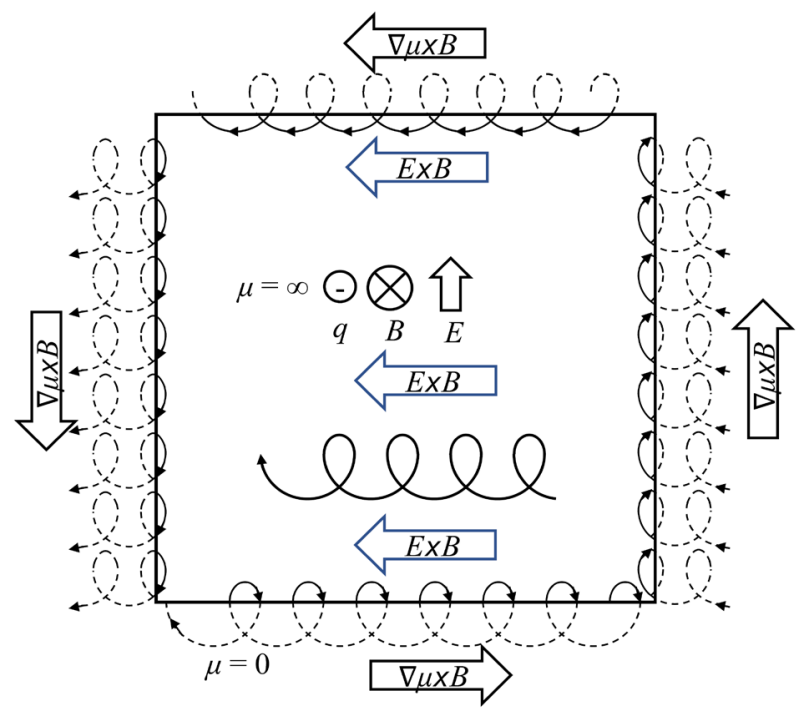

Figure 7. $\nabla \mu \times B$ and $E \times B$ drifts are enabled when a gas of electron subjected to a magnetic field and an electric field is in contact with surfaces consisting of the floor, walls and ceiling of a chamber. The drifts are parallel at the ceiling and antiparallel at the floor. 


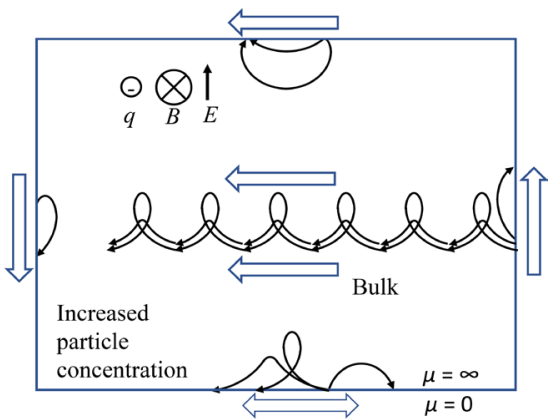

(a)

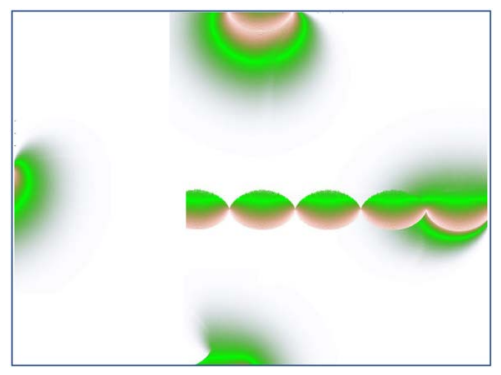

(b)

Figure 8. (a) One can easily determine by inspection that a space charge accumulates on the bottom left of the chamber. The interaction between the orbits and the surfaces depends on the orientation of the surface and the cycloid shape of the orbits. On the left wall electrons always drift down as they follow prolate cycloids. On the ceiling, they always drift left as they follow curtate or prolate cycloids. On the right wall, depending on their velocities, they either drift up the wall or leave the wall and drift through the bulk. At the floor, the drift is more complicated, but one can determine using a continuity argument that, in dynamic equilibrium, electrons move on average to the right. The drift through the bulk breaks the symmetrical circular flow, thereby increasing the concentration of particles and producing a space charge at the bottom left of the chamber; (b) This is a composite image produced by four simulations. It shows paths taken by a single particle when it leaves the floor, the ceiling or any of the walls. Blue represents the highest energy particle, green, the peak of the distribution and red, the lowest energy particles.

$\nabla \mu \times B$ drift depends on the sign of $q E$ and on the orientation of the surface (i.e., direction of $\nabla \mu$ ). In the example shown in Figure 8(a), at the floor the $E \times$ $\boldsymbol{B}$ drift points to the left, and the $\nabla \boldsymbol{\mu} \times \boldsymbol{B}$ drift, to the right and must be subtracted,

$$
v_{\text {DriftFloor }}=v_{E \times B}-v_{\mu \times B},
$$

but on the ceiling, they are in the same direction and must be added.

$$
v_{\text {DriftCeiling }}=v_{E \times B}+v_{\mu \times B} .
$$

The $\nabla \boldsymbol{\mu} \times \boldsymbol{B}$ drift (e.g., magnetic field/surface interaction) induces particles to circulate around the inside of the chamber as already illustrated in Figure 4(a) and Figure 4(b). In addition, the asymmetry introduced by the $E \times B$ drift results in the formation of space charges. This can be shown as follows. Assume that all particles in Figure 8(a) are initially uniformly distributed (no space charge) and that the number of particles $n_{\text {surface }}$ at the floor is the same as at the ceiling. From Equations (26) the current at the floor is

$$
I_{x F \text { loor }}=n_{\text {Surface }} q\left(v_{E \times B}-v_{\mu \times B}\right) \text {, }
$$

and from Equation (27), at the ceiling

$$
I_{x \text { Ceiling }}=n_{\text {Surface }} q\left(v_{E \times B}+v_{\mu \times B}\right) .
$$

Let the total number of particles in the bulk be $n_{B u l k}$ such that the bulk current is

$$
I_{x B u l k}=n_{\text {Bulk }} q v_{E \times B} .
$$


Therefore, the total initial current $I_{\text {xInitial }}$ in the $\mathrm{X}$ direction (before space charges develop) is

$$
I_{\text {xInitial }}=n_{\text {Surface }} q\left(v_{E \times B}-v_{\mu \times B}\right)+n_{\text {Surface }} q\left(v_{E \times B}+v_{\mu \times B}\right)+n_{\text {Bulk }} q v_{E \times B} .
$$

Cancelling terms:

$$
I_{\text {xInitial }}=\left(2 n_{\text {Surface }}+n_{\text {Bulk }}\right) q v_{E \times B} .
$$

Starting with uniformly distributed particles, a space charge forms downstream of the $E \times B$ drift. Eventually, equilibrium is reached which is brought upon by a shift in the $E$ field and a diffusion current caused by the charge concentration gradient. This equilibrium state can be described by adding a Fick's law [31] diffusive component to the drift equations.

\section{The Reciprocal of the Nernst Effect $(\nabla T \times B)$}

The Nernst (also called transverse or first Nernst-Ettingshausen) Effect [32] is a cross-product phenomenon: when an electrical conductor is subjected to a magnetic field $B_{z}$ and a temperature gradient $\mathrm{d} T / \mathrm{d} x$ perpendicular to the field, an electrical field $E_{y}$ is produced perpendicular to $B_{Z}$ and $\mathrm{d} T / \mathrm{d} x$.

$$
E_{y}=\mathcal{N} \frac{\mathrm{d} T}{\mathrm{~d} x} B_{z},
$$

where $\mathcal{N}$ is the Nernst constant.

Phonons moving down the temperature gradient drag electrical carriers with them, which generate a current $J_{X}$ along the $\mathrm{X}$ axis. The Hall Effect due to the magnetic field $B_{z}$, pushes the moving charges against surfaces normal to the $\mathrm{Y}$ axis thereby generating field $E_{y}$. This phenomenon, being expressed by the cross product $\nabla T \times B$, is suitable to express CPT symmetry.

The surfaces upon which charges accumulate are obstacles to electrical carriers yet must be transparent to heat phonons. In other words, electrical resistance at surfaces is required to produce charge accumulations and observable voltages. However, thermal conductivity at these surfaces is needed to permit the flow of phonons which can be measured as temperature gradients in the bulk. In more general terms, a surface can be viewed as a variation in the mobility gradient of the carriers and in the mobility gradient of the phonons, which enables the formation of a space charge and a temperature gradient.

One could conjecture a reciprocal to the Nernst effect suitable to test the second law by simply rearranging Equation (33)

$$
\frac{\mathrm{d} T}{\mathrm{~d} x}=\left(\frac{1}{\mathcal{N}}\right) \frac{E_{y}}{B_{z}}
$$

which implies that in the presence of an electric field and a magnetic field, a temperature gradient is generated perpendicular to both fields solely due to the fields, and without any input current. Comparing Equation (34) with Equation (23), one sees that this effect is simply due to the dragging of heat phonons by electrical carriers transported by the $E \times B$ current $J_{E \times B}$. This effect can be de- 
rived as follows. From Equation (23), the current $J_{E \times B}$ is

$$
J_{E \times B}=n q \frac{E_{y}}{B_{z}} .
$$

This current drags heat phonons with it, generating a heat flow along the $\mathrm{X}$ axis:

$$
\dot{Q} \propto \frac{q}{|q|} J_{E \times B}
$$

Remarkably, this heat flow is solely due to carriers dragging phonons, and contrary to Clausius' definition of the second law (heat flows from hot to cold) it is not due to any temperature gradient.

If the mobilities of the carriers and phonons are uniform along their paths (e.g., no boundary or surface), then no space charge and no temperature gradient develop. The thermal conductivity corresponding to this phonon flow is measured to be zero because $\Delta T=0$.

However, if there is no uniformity in the mobility of electrical carriers and phonons (i.e., there are gradients $\nabla \mu_{\text {carriers }}$ and $\nabla \mu_{\text {phonons }}$ ), then carriers produce space charges and local voltages (Section 3.0 The $\nabla \mu \times B+E \times B$ Drift), and phonons generate temperature gradients corresponding to a thermal conductivity $K_{\text {Phonons }}$

$$
\frac{\mathrm{d} T}{\mathrm{~d} x}=\frac{1}{K_{\text {Phonons }}} \dot{Q} .
$$

Combining Equations (35), (36) and (37) shows that a temperature gradient is produced along the $\mathrm{X}$ axis

$$
\frac{\mathrm{d} T}{\mathrm{~d} x} \propto \frac{n|q|}{K_{\text {Phonons }}} \frac{E_{y}}{B_{z}} .
$$

Defining $1 / \mathcal{N}$ as the constant of proportionality, one obtains the reciprocal Nernst effect as conjectured in Equation (34).

$$
\frac{\mathrm{d} T}{\mathrm{~d} x}=\left(\frac{1}{\mathcal{N}}\right) \frac{E_{y}}{B_{z}} .
$$

The temperature gradient is generated in the direction of $J_{E \times B}$, perpendicularly to $B_{z}$ and $E_{y}$. Therefore, the reciprocal Nernst Effect is a cross-product phenomenon, one of the requirements for testing the second law as stated in the Introduction.

The temperature gradient is caused by the accumulation of heat phonons carried by electrical carriers circulating under the influence of the mobility gradient and $E \times B$ drift. It occurs spontaneously, driven by thermal energy drawn from thermalizing surfaces.

One should note that the effect described in this section requires a dynamic system that supports the continuous and spontaneous circulation of gas particles, as described in Sections 2 and 3. Simulation of this system shows that gas particles move in a conveyor belt fashion, going to the left in the bulk and to the 
right along the floor of the simulation chamber as shown in Figure 7, Figure 8, Figure 10 and Figure 11. A static system would be incapable of producing a temperature gradient as it would revert to the Loschmidt temperature paradox explained under Section 6.1.2 Spontaneous Temperature Gradients by Scalar Fields. In a static equilibrium, no temperature gradient can arise.

This effect would never have been approved by Onsager who excluded magnetic fields and rotation from his reciprocals because of a conflict with the second law. His exclusion resolves the conflict but ends up violating CPT symmetry. In the opinion of this author, he was incorrect in his reasoning (see explanation in Appendix B) and threw the baby out with the bathwater. In any case, by upholding CPT symmetry, one can turn his argument on its head and take it as a proof that magnetic fields and rotation can produce a second law violation.

\section{Simulations}

An $E \times B$ drift was simulated for a low-density electron gas subjected to a magnetic field $B_{z}$ and an electric field $E_{y}$. The position of the electrons was displayed as projected on the $\mathrm{XY}$ plane.

Electrons were initialized in the simulation chamber at random positions and with a maxwellian velocity distribution. They followed cycloid paths which, depending on the initial velocity $v_{x y}$ and the magnitude of the $E$ and $B$ fields, could either be curtate, regular, or prolate cycloids as shown in Figures 9(a)-(d).

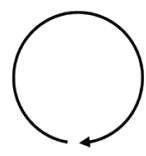

Circle: $\frac{E_{y}}{B_{z}}=v_{E \times B}=0$

(a)

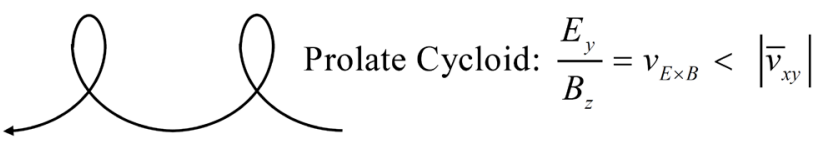

(b)

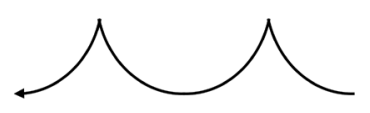

Cycloid: $\frac{E_{y}}{B_{z}}=v_{E \times B}=\left|\bar{v}_{x y}\right|$

(c)

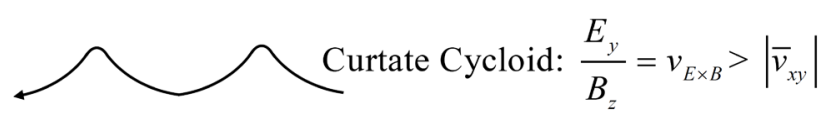

(d)

Figure 9. (a) When the electric field is zero, the drift velocity is zero and the orbit is circular; (b) When the drift velocity $v_{E \times B}$ is smaller than the particle velocity $v_{x y}$ in the $\mathrm{XY}$ plane, then the trajectory is a prolate cycloid; (c) When $v_{E \times B}=v_{x y}$, then the trajectory is a cycloid; (d) When $v_{E \times B}>v_{x y}$, then the trajectory is a curtate cycloid. The shape of these cycloids determines how a particle interacts with a surface i.e., drifts up, down, right or left. 
The output of the simulation program, shown in Figures 10(a)-(d) and Figure 11. This last figure clearly indicates the accumulation of particles (space charge) at the bottom left of the simulation chamber.

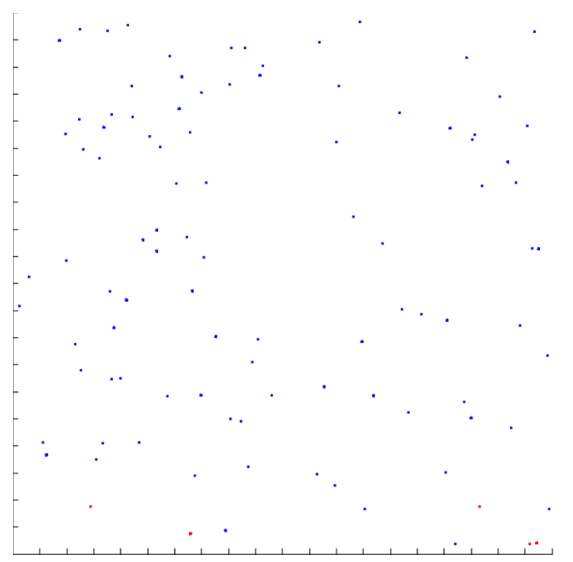

(a)

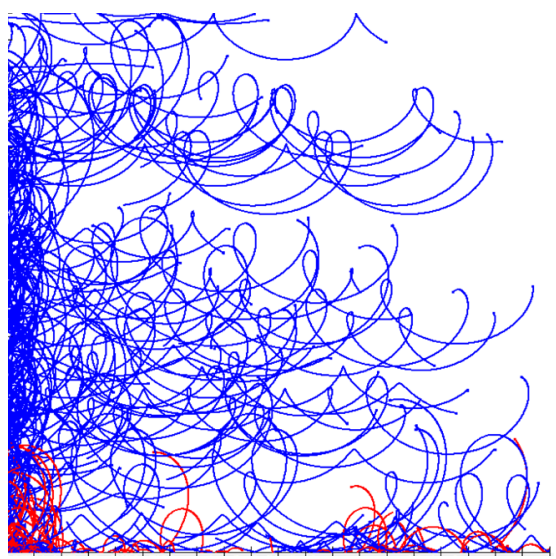

(a)

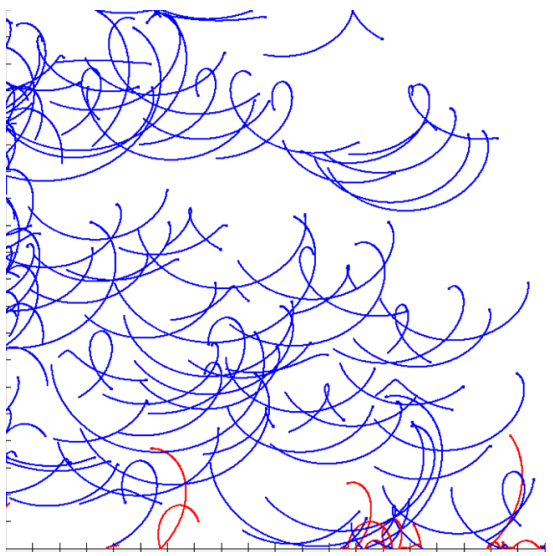

(b)

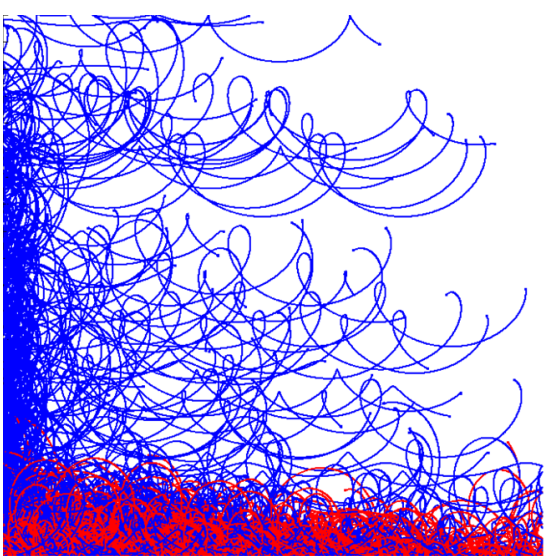

(b)

Figure 10. Four frames of a time lapse simulation showing particle trajectories as (a) they begin randomly distributed throughout the chamber; (b) they are carried by the $E \times B$ drift toward the left wall; (c) they accumulate on the left wall; (d) they accumulate on the floor in the bottom left corner. Red trajectories represent particles moving right, and blue trajectories, particles moving left, up or down.

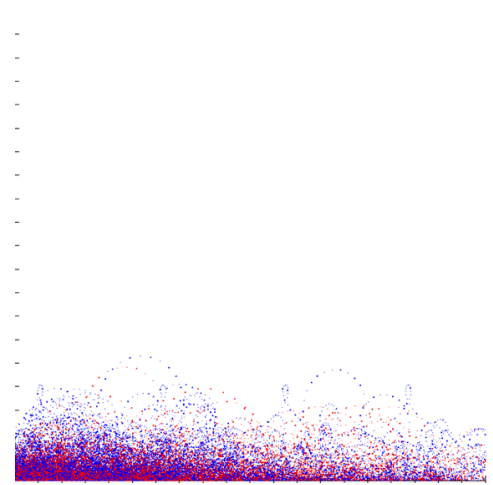

Figure 11. This figure displays the positions of particles after equilibrium is reached. The concentration is highest at the bottom left corner. 


\section{The Second Law-Broken or Bypassed?}

Three phenomena that incorporate CPT symmetry and collisions through a cross-product have been presented.

1) The $\nabla \boldsymbol{\mu} \times \boldsymbol{B}$ drift transfers momentum between a surface and the orbital motion of charged particles, thereby causing the gas or particles to circulate in a direction opposite to their orbits.

2) The combination $\nabla \boldsymbol{\mu} \times \boldsymbol{B}+\boldsymbol{E} \times \boldsymbol{B}$ drift causes particles to accumulate into space charges and produce voltages.

3) The reciprocal Nernst effect produces a temperature gradient without the need of heat flow or current, solely by means of $\boldsymbol{E}$ and $\boldsymbol{B}$.

Can these effects convert ambient heat to useful energy? Before answering this question, one needs to recognize that the field of second law violations is treacherous, replete with hidden traps and failed Maxwell demons. Therefore, in proceeding with caution the following shall be discussed:

1) Review of most relevant second law pitfalls.

2) Getting around the law.

\subsection{Review of Most Relevant Second Law Pitfalls}

This section includes a short summary of some failed Maxwell Demons selected because like the approaches outlined in the previous sections, they use fields in their attempts to produce a voltage or a temperature gradient. The discussion also covers how these pitfalls differ from the suggested approach and includes:

1) Spontaneous potentials by scalar fields.

2) Spontaneous temperature gradients by scalar fields.

3) Spontaneous temperature gradients by quantum statistics.

\subsubsection{Spontaneous Potentials by Scalar Fields}

The built-in voltage in a diode junction is sometime suggested as an energy source. It is inaccessible because it is exactly canceled by the potentials produced by the contacts. Other instances of inaccessible voltages are the work function and the chemical and surface potentials of materials.

For the same reason, the difference in pressures between two points at different depths in the ocean cannot be used to produce energy. Any pipe connecting the two points, say to run a turbine, develops the same pressure difference and the turbine does not work. Fundamentally, this limitation is due to the scalar property of the (electric or gravitational) field. Any loop integral of potential differences must necessarily be zero.

\subsubsection{Spontaneous Temperature Gradients by Scalar Fields}

The typical example of this second law trap is the Loschmidt's temperature gradient paradox [4]. He mistakenly believed that a column of Maxwellian gas would spontaneously (adiabatically) develop a temperature gradient as the gas particles move up and down the gravitational field. Particles that diffuse upward convert kinetic energy to potential energy, and particles which diffuse downward, 
do the reverse. This temperature gradient would then be available to run a heat engine, thereby converting ambient heat to useful work. In fact, the column remains isothermal for the following reason. In a classical gas, energy is expressed exponentially in a Maxwell distribution

$$
f_{M B}(E, T)=A \mathrm{e}^{-\frac{E}{k_{B} T}} .
$$

A rise in elevation causes an increase in the potential energy term $E_{p}$ expressed in the exponent.

$$
f_{M B}(E, T)=A \mathrm{e}^{-\frac{E+E_{p}}{k_{B} T}} .
$$

It also results in a decrease in the gas density accompanied by a lowering of the amplitude and a denormalization of the distribution. This decrease in amplitude can be expressed by factoring potential energy out of the exponent.

$$
f_{M B}(E, T)=A \mathrm{e}^{-\frac{E_{p}}{k_{B} T}} \mathrm{e}^{-\frac{E}{k_{B} T}} .
$$

Renormalization to correct the amplitude term, removes the potential energy factor thereby restoring the distribution to its original ground level form as expressed in Equation (40). Therefore, the normalized distribution is invariant with elevation, and the column remains isothermal. This second law trap applies to any situation in which a scalar force acts on classical particles [33].

\subsubsection{Spontaneous Temperature Gradients by Quantum Statistics}

Loschmidt's classical temperature paradox discussed above can be extended to quantum distributions. Consider a gas column comprised of fermions in a force field. As in a Maxwell-Boltzmann case, energy is expressed in the exponential part of the Fermi-Dirac distribution.

$$
f_{F D}(E, T)=\frac{A}{1+\mathrm{e}^{\frac{E}{k_{B} T}}} .
$$

A rise in elevation causes an increase in potential energy in the exponent

$$
f_{F D}(E, T)=\frac{A}{1+\mathrm{e}^{\frac{E+E_{p}}{k_{B} T}}} .
$$

However, renormalization does not restore the distribution to its ground level form because the distribution is not purely exponential. Therefore, (this is the trap) the distribution is not invariant, implying that the kinetic energy of the gas particle does vary with elevation (which is correct). Yet, the effective thermodynamic temperature of the gas is invariant for the following reason.

The Fermi-Dirac distribution includes "frozen out" microstates occupied by particles at the bottom of the Fermi sea, and "unfrozen" microstates occupied by particles near the surface of the sea. The frozen microstates are not thermodynamically active, leaving only the unfrozen ones available for processes such as thermal transport and thermal capacity. The effective temperature depends on 
the average kinetic energy of the unfrozen microstates, not the average kinetic of all microstates. The statistics of the unfrozen microstates are described by the exponential part of the Fermi-Dirac distribution, not the whole distribution, in other words, the distribution of unfrozen microstates is Maxwellian. These states cannot spontaneously produce a temperature gradient for the same reasons given in the previous section on the Loschmidt's temperature gradient paradox. Therefore, temperature is isothermal up and down the column in accordance with the second law. See Note [34]). This same phenomenon is responsible for the depression of specific heat from the classical Dulong-Petit form to the Debye/Einstein form.

\subsection{Getting Around the Law}

CPT systems use a magnetic field which is non-scalar. Therefore, they avoid the scalar field pitfall outlined in Section 6.1.1. They have asymmetrical non Maxwellian statistics, therefore they avoid the pitfalls outlined in Sections 6.1.2 and 6.1.3.

Is the second law violated by CPT symmetric systems? Surprisingly, if one were to use Boltzmann's definition of the law, the short answer is no.

To answer this question properly, one needs to define the second law. As Čápek and Sheehan [35] remark, this law has been differently formulated by researchers such as Carnot, Clausius, Plank, Kelvin, Caratheodory, Gibbs and Boltzmann.

Some formulations are empirical or experimentally inductive such as heat flows from hot to cold, (Clausius,) and perpetual motion machines are impossible (Kelvin-Planck). Induction, however, is not reliable as Bertrand Russell demonstrates with his farmer/chicken paradox. The chicken, being fed daily by the farmer, induces that he is a benefactor. Yet one day the farmer wrings the animal's neck. These formulations, in Popper's words, are experimentally falsifiable.

In contrast, Boltzmann's second law, the H-Theorem, is deductive and rigorously derived. It correctly proves that entropy never decreases in an isolated gas. Yet, this rigor hides a weakness predicated by the assumptions made at the outset of the deductive process which, for the H-Theorem [2] [36] [37] are:

1) The gas is homogeneously distributed.

2) The statistics are symmetrical.

3) The gas particles are indistinguishable (Gibbs paradox [6]).

4) Time is unidirectional (arrow of time).

The H-Theorem is deductive and therefore limited by its starting assumptions. Any system falling outside of the box defined by these assumptions is not limited by, and may not comply with, the theorem. Yet the theorem remains logically consistent within its range of coverage.

The CPT systems discussed in this paper do not comply with the assumptions of the H-Theorem because:

1) The gas is not homogeneously distributed. Heat carriers including electrical 
carriers and phonons, are heterogeneously distributed throughout the system. Phonons get around the scalar limitation of the electrical field because they are neutral bosons, not affected by the fields. A loop integral of potential differences through these carriers is not zero.

2) CPT symmetric systems have an asymmetrical velocity distribution. In a magnetic field, the interaction of particles with the surface skews the position and velocity distributions. This effect was unanticipated by Boltzmann in his H-Theorem.

3) The gas particles comprise electrically charged carriers and neutral phonons which are distinguishable by their charges, spin and mass, and by their heterogeneous distributions.

In summary, CPT symmetric systems fall outside of the starting assumptions of the H-Theorem. They do not "break" the second law as defined by Boltzmann, they simply "bypass" it.

\section{Experimental Evidence Supporting the Theory}

$\mathrm{Fu}$ and $\mathrm{Fu}[28]$ conducted experiments in which they applied a magnetic field to two identical and parallel Ag-O-C surfaces in a vacuum tube at room temperature. A current was produced from one surface to the other, in a direction dependent on the field's direction. Their work, which mostly replicates the Crookes radiometer experiment suggested in Section 2.3 and elaborated upon in Section 9, confirms the theory proposed in this paper.

Further evidence involves mysterious spurious voltages often and reliably observed in the course of plasma $\boldsymbol{E} \times \boldsymbol{B}$ drift experiments by independent scientists. Did the researchers unwittingly violate the second law by drawing energy through their instrument in the act of collecting data? Possibly. The significance of these voltages may have been masked by the high plasma temperatures which preclude energy production [29] [30]. In any case, a definite answer to this question can only be provided by follow up experiments.

\section{Conclusions}

CPT symmetrical systems in which parity is expressed by a cross product between the magnetic field and the mobility gradient (a measure of dissipation) fall outside assumptions in the $\mathrm{H}$-Theorem because these systems skew statistics in a fashion unanticipated by the founders of thermodynamics. The proof of the theorem remains valid within its domain of applicability; therefore, these systems do not break the second law, they bypass it.

Three kinds of systems were discussed. The first ones which combine the mobility gradient with the magnetic field $(\nabla \mu \times B)$ produce spontaneous circulation of gas particles inside a chamber. This movement is driven by thermal energy from the wall of the chamber. The second ones which include an electric field $(\nabla \mu \times B+E \times B)$ generate spontaneous space charges and voltages. The third ones which embody the reciprocal of the Nernst Effect $(\nabla T \times B)$ use cir- 
culating electrical carriers to produce spontaneous temperature gradients.

A simulation has been performed that confirms the theory. The proposed magnetic Crookes' experiment has already essentially been conducted [28] and confirms the theory. In addition, experimental data unintentionally collected in $\boldsymbol{E} \times \boldsymbol{B}$ experiments by several independent researchers indicate that such phenomena can produce spontaneous space charges and voltages.

Non-scalar fields such as the magnetic field in conjunction with a mobility gradient can impart circulation to heat flow, implying that temperature and entropy can have circulation as well. This represents a direct challenge to the zeroth law and to the principle of detailed balance. This topic should be further explored.

Laboratory experiments are suggested to further validate or falsify the proposed technology. Its benefit cannot be overstated: conversion of ambient heat to useful energy, the only by-product being cold; unlimited non-polluting renewable green energy. Given the energy production potential of the proposed technology, future research should concentrate on physical configurations that operate at or below room temperature, including thermoelectric and superconductor implementations.

\section{Suggested Future Research}

Given its potential as an energy source, this technology should be experimentally validated or falsified. Several laboratory conditions should be investigated:

1) Plasma. $E \times B$ drift experiments should be repeated for the express purpose of testing the second law. Even though the high operating temperatures of the plasma negate any economic value, this approach is easy to implement and has scientific merit because it shows that, regardless of temperature, the second law can, in principle, be bypassed.

2) Magnetic Crookes' radiometer. Such a radiometer comprises an opaque vacuum bulb placed in a vertical magnetic field, containing fixed vanes, and coated on the inside with a low work function material such as Lanthanum Hexaboride to emit (and absorb) electrons at a high enough temperature. Each vane is configured as a capacitor comprised of an insulating plate in a sandwich between two conductive films. Circulating electrons would then produce an $a b$ servable voltage across the faces of the stationary vanes. This experiment has already been essentially performed by $\mathrm{Fu}$ and $\mathrm{Fu}$ [28] but should be replicated and confirmed.

3) Thermoelectric materials. Electrical carriers (electrons and holes) in high mobility materials such as Indium Antimonide (InSb) can undergo $\boldsymbol{E} \times \boldsymbol{B}$ drift. and therefore, provide attractive room-temperature implementation for the proposed technology.

4) Superconductors. Carriers in superconductors (Cooper pairs) behave as a gas and therefore, can also support the proposed technology. Since the device is a superconductor, it would have to be thin to operate with a magnetic field. As 
the depletion mode in semiconductors allows the presence of an electric field, the London depth region in superconductors allows the penetration of the magnetic field which is necessary for operation. Alternatively, type II or type 1.5 superconductors could be used. This approach could be highly efficient. Since superconducting devices operate at low temperature, ambient heat at surrounding room temperature would rapidly "fall" into them (i.e., thermal black holes analogy.) Converting this heat to electricity would result in self-sustained highly efficient superconducting devices.

\section{Supplementary Materials}

The computer code used in the simulations can be provided upon request.

\section{Acknowledgements}

I would like to thank my wife Penny for her unwavering support, and Daniel Sheehan for his invaluable guidance and advice.

\section{Conflicts of Interest}

The author declares no conflicts of interest regarding the publication of this paper.

\section{References}

[1] Albert Einstein, Wikiquote. https://en.wikiquote.org/wiki/Albert_Einstein

[2] Boltzmann, L. (1872) Weitere Studien über das Wärmegleichgewicht unter Gasmolekülen. Sitzungsberichte Akademie der Wissenschaften, 66, 275-370. [English Translation: Boltzmann, L. (2003) Further Studies on the Thermal Equilibrium of Gas Molecules, The Kinetic Theory of Gases. History of Modern Physical Sciences, 1, 262-349.] https://doi.org/10.1142/9781848161337_0015

[3] Loschmidt's Time Reversibility Paradox, Wikipedia. https://en.wikipedia.org/wiki/Loschmidt's_paradox

[4] Andreas, T. (1999) Energy, Entropy: On the Occasion of the 100th Anniversary of Loschmidt's Death in 1895: Is Loschmidt's Greatest Discovery Waiting for Its Discovery? Physics Essays, 12, 614-628.

[5] Arrow of Time, Wikipedia. https://en.wikipedia.org/wiki/Arrow_of_time

[6] Gibbs Paradox, Wikipedia. https://en.wikipedia.org/wiki/Gibbs_paradox

[7] Gibbs, J.W. (2019) On the Equilibrium of Heterogeneous Substances. https://radarmet.atmos.colostate.edu/AT620/papers/Gibbs1875-1878-Equilibrium of_Heterogeneous_Substances.pdf

[8] Tolman, R.C. (1938) The Principle of Statistical Mechanics. Clarendon Press, Oxford.

https://archive.org/details/ThePrinciplesOfStatisticalMechanicsTolmanOxfordAtTh eClarendonPress1938

[9] Von Neumann, J. (1929) Proof of the Ergodic Theorem and the H-Theorem in Quantum Mechanics. arXiv:1003.2133v2.

[10] Von Neumann, J. (1955) Mathematical Foundations of Quantum Mechanics. Princeton University Press, Princeton, NJ. 
[11] Evans, D.J., Cohen, E.G.D. and Morriss, G.P. (1993) Probability of Second Law Violation in Shearing Steady States. Physical Review Letters, 71, 2401-2404. https://pdfs.semanticscholar.org/697a/31eace27d3b0096700709d83d5f3bd8f1733.pdf

[12] Evans. D.J. and Searles, D.J. (1996) Causality, Response Theory, and the Second Law of Thermodynamics. Physical Review E, 53, 5808. https://doi.org/10.1103/PhysRevE.53.5808

[13] Evans D.J. and Searles, D.J. (2002) The Fluctuation Theorem. Advances in Physics, 51, 1529-1585. https://pdfs.semanticscholar.org/470a/4e148c2ba7aa0f6d48d455d9a854db2a1da2.pdf

[14] Klimenko, A.Y. (2017) Kinetics of Interactions of Matter, Antimatter and Radiation Consistent with Antisymmetric (CPT-Invariant) Thermodynamics. Entropy, 19, 202. https://doi.org/10.3390/e19050202

[15] Klimenko, A.Y. (2016) Symmetric and Antisymmetric Forms of the Pauli Master Equation. Scientific Reports, 6, 29942. https://doi.org/10.1038/srep29942

[16] Klimenko, A.Y. and Maas, U. (2014) One Antimatter-Two Possible Thermodynamics. Entropy, 16, 1191-1210. https://doi.org/10.3390/e16031191

[17] Onsager, L. (1931) Reciprocal Relationships in Irreversible Processes. I. Physical Review Journals Archive, 37, 407-425. https://doi.org/10.1103/PhysRev.37.405

[18] Onsager, L. (1931) Reciprocal Relationships in Irreversible Processes. II. Physical Review Journals Archive, 38, 2265. https://doi.org/10.1103/PhysRev.38.2265

[19] Note: The mobility gradient effect may be familiar to anyone who has driven in winter on a slippery road, lightly covered with snow in the center (because of traffic), and more heavily, near the shoulder. Driving slightly off center, say to the right, increases drag on the right wheels, producing a torque that makes the car veer right, thereby producing more drag on the right wheels. A force is generated perpendicularly to the direction of motion and along the mobility gradient, causing the car to drift quickly and uncontrollably off the icy road and into the snowbank. In this case, there is no magnetic field and the resulting motion occurs along this force.

[20] Levy, G.S. (2017) The Reciprocal Hall Effect, CPT Symmetry and the Second Law. Open Science, 4, 1-8.

http://www.openscienceonline.com/journal/archive2?journalId=725\&paperId=3723

[21] Levy, G.S. (2017) Choosing between the Reciprocal Hall Effect, CPT symmetry and the Second Law. The Open Science Journal of Modern Physics, 4, 1-8.

https://www.researchgate.net/publication/314899020

[22] Crooks, G.E. (1999) The Entropy Production Fluctuation Theorem and the Nonequilibrium Work Relation for Free Energy Differences. Physical Review E, 60, 2721. https://doi.org/10.1103/PhysRevE.60.2721

[23] Duncan, T.L. (2000) Comment on "Dynamically Maintained Steady-State Pressure Gradients”. Physical Review E, 61, 4661. https://doi.org/10.1103/PhysRevE.61.4661

[24] Thermodynamic Limit, Wikipedia. https://en.wikipedia.org/wiki/Thermodynamic_limit

[25] Sheehan, D.P. and Gross, D.H.E. (2006) Extensivity and the Thermodynamic Limit: Why Size Really Does Matter. Physica A: Statistical Mechanics and Its Applications, 370, 461-482.

https://www.researchgate.net/publication/223328200_Extensivity_and_the_thermo dynamic_limit_Why_size_really_does_matter https://doi.org/10.1016/j.physa.2006.07.020 
[26] Indium Antimonide, Wikipedia. https://en.wikipedia.org/wiki/Indium_antimonide

[27] Guiding Center, Wikipedia. https://en.wikipedia.org/wiki/Guiding_center

[28] Fu, X. and Fu, Z. (20106) Realization of Maxwell's Hypothesis: A Heat-Electric Conversion in Contradiction to the Kelvin Statement. https://doi.org/10.20944/preprints201607.0028.v5

[29] Motley, R.W. (1975) Q-Machines. Academic Press, New York.

[30] Rynn, N. and D’Angelo, N. (1960) Device for Generating a Low Temperature, Highly Ionized Cesium Plasma. Review of Scientific Instruments, 31, 1326. https://doi.org/10.1063/1.1716884

[31] Fick's Law of Diffusion, Wikipedia. https://en.wikipedia.org/wiki/Fick\%27s_laws_of_diffusion

[32] Nernst Effect, Wikipedia. https://en.wikipedia.org/wiki/Nernst_effect

[33] Kollasch, J. (2014) Private Communication.

[34] Note: The author acknowledges that in previous publications [43] [44] [45] [46] he relied on the skewing of distributions by quantum statistics and takes this opportunity to correct the record. The quantum statistical examples he used in these papers do not skew the distribution of thermal carriers (unfrozen microstates). However, the CPT symmetrical systems discussed in this paper do. All previously published results relying on statistical skewing still stand when applied to CPT symmetric systems. Asymmetrical statistics can bypass the second law, temperature gradients can form spontaneously. Heat flow in the presence of a magnetic field can have circulation implying that temperature and entropy may not be scalar.

[35] Čápek, V. and Sheehan, D. (2005) Challenges to the Second Law of Thermodynamics. Springer, New York. https://doi.org/10.1007/1-4020-3016-9

[36] Uffink, J. (2006) Compendium of the Foundations of Classical Statistical Physics. 44-45. http://philsci-archive.pitt.edu/2691/1/UffinkFinal.pdf

[37] Brown, H. and Myrvold, W. (2008) PhilSci-Archive. http://www.socsci.uci.edu/ bskyrms/bio/readings/brown_myrvold_on_boltzmann.pdf http://philsci-archive.pitt.edu/4187/1/Reversibility08.pdf

[38] Crooks, G.E. (1999) The Entropy Production Fluctuation Theorem and the Nonequilibrium Work Relation for Free Energy Differences. Physical Review E, 60, 2721.

[39] Mazur, P. and Degroot, S.R. (1953) On Onsager's Relations in a Magnetic Field. Physica, 19, 961-970. https://doi.org/10.1016/S0031-8914(53)80108-4

[40] Trigonometric Integrals, Wikipedia. https://en.wikipedia.org/wiki/Trigonometric_integral

[41] Entropic Force, Wikipedia. https://en.wikipedia.org/wiki/Entropic_force

[42] Drude Model, Wikipedia. https://en.wikipedia.org/wiki/Drude_model

[43] Levy, G.S. (2018) Using Quantum Statistics to Win at Thermodynamics, and Cheating in Vegas. Journal of Applied Mathematics and Physics, 6, 2166-2179. https://www.researchgate.net/publication/328605676_Using_Quantum_Statistics_to _Win_at_Thermodynamics_and_Cheating_in_Vegas

[44] Levy, G.S. (2018) Temperature and Voltage Offsets in High ZT Thermoelectrics. Journal of Electronic Materials, 47, 3067-3076. https://doi.org/10.1007/s11664-017-5875-0

[45] Levy, G.S. (2015) Quantum Game Beats Classical Odds-Thermodynamics Implications. Entropy, 17, 7645-7657. https://doi.org/10.3390/e17117645 
[46] Levy, G.S. (2016) Anomalous Temperature Gradient in Non-Maxwellian Gases. In: Ceramics for Energy Conversion, Storage, and Distribution Systems. Ceramic Transactions, Volume 255, Wiley, Hoboken, NJ.

https://doi.org/10.1002/9781119234531.ch11 


\section{Appendix A. The Fluctuation Theorem Assumes the Arrow of Time}

One of the most recent contributions to the time reversibility paradox is the fluctuation theorem first proven by Evan, Cohen and Morriss [11] in 1993. This theorem is, in fact, a group of closely related theorems, two of which shall be discussed. The one by Evans and Searles [11] [12] [13] describes the behavior of the time-average entropy production rate $\bar{\Sigma}_{t}$ in terms of probability. It states [13] that the ratio of the probability that $\bar{\Sigma}_{t}=A$ to the probability that $\bar{\Sigma}_{t}=-A$ can be expressed as an exponential function of time.

$$
\frac{\operatorname{Pr}\left(\bar{\Sigma}_{t}=A\right)}{\operatorname{Pr}\left(\bar{\Sigma}_{t}=-A\right)}=\mathrm{e}^{A t}
$$

In other words, as time $t$ elapses or system size $A$ increases, the probability of observing an occurrence of entropy production opposite to the second law decreases.

The second theorem is due to Crooks [38]. It relates the probability of a space-time trajectory $x(t)$ to the probability of its time-reversal trajectory $\tilde{x}(t)$. It states that the forward time trajectory is exponentially more likely than the reverse, given that it produces entropy $\omega[x(t)]$,

$$
\frac{P[x(t)]}{P[\tilde{x}(t)]}=\mathrm{e}^{+\omega[x(t)]} .
$$

Both these theorems co-opt the very foundation of Loschmidt's argument by relying on the assumption of time reversibility. However, precisely because the time parameter $t$ is expressed symmetrically as shown in Equations (45) and (46), they are unable by themselves to resolve Loschmidt's paradox in favor of the second law. As Evans and Searles point out, inverting the time axis, i.e., $t \rightarrow-t$ produces a decrease in entropy, an anti-second law result.

Evans and Searles [13] attempt to resolve this issue by classifying systems as causal (those in which cause precedes effect) and anticausal (those in which effect precedes cause). Entropy increases in causal systems when time flows forward, and in anticausal systems when time flows backward. An observer in a backward moving time frame would think that an anticausal system is in fact causal because in his frame, he would perceive his cause (our effect) to precede his effect (our cause). He would observe an increase in entropy in his system, yet a second observer in a forward moving frame would observe a decrease in the entropy of the system of the first observer.

Evans and Searles acknowledge the problem and seem to agree with Loschmidt (page 1580 of [13]):

"If every microscopic law or axiom of Nature is symmetric under time reversal symmetry, then obviously one cannot derive time asymmetric theorems such as the FTs'.

In the remainder of this quote they suggest a solution:

"Somewhere in the derivations of the FT given in Section 2.3, we must have 
introduced a time asymmetric assumption. That assumption was the Axiom of Causality".

They state (Abstract of [13]):

"It is causality which ultimately is responsible for breaking time reversal symmetry and which leads to the possibility of irreversible macroscopic behavior".

They resolve the issue by showing that the fluctuation theorem in combination with the axiom of causality proves the second law. Their solution bypasses Loschmidt's paradox by negating its basic premise: the time reversibility assumption. Stepping outside the box defined by Loschmidt, they assert that time is not symmetrical after all, it only moves forward, the arrow of time points in the positive direction and anticausal systems are not physical.

Yet the issue is not fully resolved. A backward moving observer would come to the opposite conclusion. For him, time would flow in the opposite direction and he would ban causal systems from his physics. We have no way of knowing which observer we are, and in which direction time really flows. Nevertheless, just like Boltzmann failed to do in his H-Theorem, they do not explain the arrow of time. They assume it.

Another serious issue is that the axiom of causality as defined by Evans and Searles contradicts locality. They describe a causal system (their equation 6.4a in [13]) as

$$
B_{C}(t)=\int_{-\infty}^{t} L_{C}\left(t-t_{1}\right) F\left(t_{1}\right) \mathrm{d} t_{1},
$$

where $L_{C}$ is the response function, $F$ is a driving force and $B_{C}$ is the system's response. One should note that the integral is taken from $-\infty<t_{1}<0$ which implies that the states of causal system are defined by its (long ago) non-local past.

This definition violates locality. To fix this problem, one would have to recognize that an event long ago, far away within a light cone, is only relevant to the present if a causal chain exists between this event and the present. If one were to retain only the most recent relevant history up to time-d $t$, then Equation (47) could be expressed differentially:

$$
\mathrm{d} B_{C}(t)=\int_{t-\mathrm{d} t}^{t} L_{C}\left(t-t_{1}\right) F\left(t_{1}\right) \mathrm{d} t_{1}=L_{C}(\mathrm{~d} t) F(t) \mathrm{d} t,
$$

or

$$
\frac{\mathrm{d} B_{C}(t)}{\mathrm{d} t}=L_{C}(0) F(t) \mathrm{d} t .
$$

This equation respects strict locality. It can be rendered as a geometrical construct devoid of any cause/effect relationship, thereby nullifying the axiom of causality and bringing us back to the need for the arrow of time postulate.

\section{Appendix B. CPT Symmetry and the Second Law-Onsager's Dilemma}

Onsager's reciprocals are thermodynamic relationships between pairs of forces 
and flows. For example, the Seebeck is the generation of a voltage in the presence of a temperature difference and the Peltier effect is the production of heat flow by an electric current. Onsager was concerned about CT symmetry such as the magnetic field and rotation. He excluded them from his reciprocals to avoid a second law violation. This appendix discusses this exclusion. A quick overview of his paper [17] is presented, which includes his derivation and the conflict with the second law. Accordingly, this appendix includes:

1) Derivation of reciprocals.

2) Conflict with the second law.

3) Hidden assumptions: i.e., surfaces.

\section{B.1. Derivation of Reciprocals}

Onsager's derivation relies on the assumption of time $(T)$ reversibility of microscopic fluctuations. It begins by expressing the phenomenological relationship $J$ $=\boldsymbol{L} \boldsymbol{X}$ between thermodynamic forces $\boldsymbol{X}$ and thermodynamic fluxes $\boldsymbol{J}$. Dropping the vector notation, the relationship can be expressed as

$$
J_{k}=\sum_{j} L_{k j} X_{j} .
$$

where, as an example, $X_{k}=(\mathrm{d} T / \mathrm{d} x) / T$ and $J_{k}=\mathrm{d} Q / \mathrm{d} t$. There is no need to go into the details of the proof here as it is available in [17]. The result relates three properties:

1) Magnetic field and rotation;

2) T-reversibility of microscopic fluctuations, and;

3) The symmetry of the phenomenology relationship matrix $L$. This symmetry is expressed as $L_{k j}=L_{k j}$.

The proof makes the forward inference very clear:

No Magnetic Field AND No Rotation $\rightarrow T$-Reversible $\rightarrow L$ Symmetric

Using modus tollens, produces.

Magnetic Field OR Rotation $\leftarrow$ Not $T$-Reversible $\leftarrow L$ Asymmetric

However, Onsager needs the converse inference to justify his exclusion.

Magnetic Field OR Rotation $\rightarrow$ Not $T$-Reversible $\rightarrow L$ Asymmetric.

On this point, the proof is questionable as shall be discussed in the third section of this appendix that covers hidden assumptions made by Onsager in his proof. For the moment, let us accept this converse inference and continue his reasoning.

Onsager could have accepted Equation (53) at face value and extended reciprocals to magnetic and rotating environments. He could have done so, by splitting the $L$ matrix into symmetric and antisymmetric parts, the symmetric part of $L$ being applicable to non-magnetic environments, and the antisymmetric part, to magnetic ones. This approach is analogous to splitting an electromagnetic phenomenon into an electric field and a magnetic field.

This is precisely the approach taken by Mazur and de Groot [39] who show 
that the phenomenological matrix $L$ can be antisymmetric. However, Onsager did not take this route because of the apparent conflict with the second law described below.

\section{B.2. Conflict with the Second Law}

This proof uses the second law to show that the phenomenological relation matrix $L$ must be symmetric. The rate of entropy production can be expressed in terms of $\boldsymbol{J}$ and $\boldsymbol{X}$ as

$$
\frac{\mathrm{d} S}{\mathrm{~d} t}=\sum_{k} J_{k} X_{k} .
$$

The second law can be stated as $d S / d t>0$.

$$
\frac{\mathrm{d} S}{\mathrm{~d} t}=\sum_{k} J_{k} X_{k}>0 \text {. }
$$

Combining with Equation (50) one gets:

$$
\sum_{j k} L_{k j} X_{k} X_{j}>0 .
$$

Therefore, asserting the second law is the same as stating that $L$ is positive definite. One knows from matrix algebra that a positive definite matrix must be symmetric and all its eigenvalues, positive. Furthermore, an asymmetric matrix cannot be positive definite. Therefore, Onsager's second derivation can be stated as:

Second Law Violated $\leftrightarrow L$ Not Positive Definite $\leftarrow L$ Asymmetric. (57)

Equation (53) indicates that a magnetic field or rotation causes $L$ to be asymmetric. Furthermore, Equation (57) states that if $L$ is asymmetric then the second law is violated. Combining the two equations one concludes that magnetic fields or rotations violate the second law.

Faced with this daunting violation, Onsager's response was simple. He restricted magnetic fields and rotation from reciprocals, thereby excluding a wide range of phenomena, without addressing the heart of the problem which was the conflict between the second law and CT symmetry.

In the last two paragraphs of his paper (page 426 [17]) he appears to have had second thoughts, making the vague argument that reciprocal symmetry can be preserved by simply flipping the magnetic field and reversing time.

"In the presence of a magnetic field the principle of microscopic reversibility may be applied in a modified form: The entire motion may be reversed by reversing the magnetic field together with the velocity of all the particles composing the dynamical system".

By suggesting "reversing the magnetic field" to reverse the velocity of all particles, he departs from the basic premise of his proof which is the time reversibility of microscopic fluctuations. His suggestion does not work because:

1) An externally applied DC magnetic field does not fluctuate enough to reverse particle velocities. 
2) Charges are conserved, they do not fluctuate.

His attitude towards CT symmetric systems is ambivalent. He argues that they may still be included in his reciprocals, but still leaves them out without answering whether they violate the second law. Nevertheless, this exclusion is the equivalent of a sweep under the carpet. It leaves the conflict in a stalemate.

\section{B.3. Hidden Assumption: i.e., Surfaces}

Onsager's assumes the reversibility of microscopic fluctuations. This assumption precludes any change in mobility at the microscopic level. For example, surfaces are excluded. Using the term "surface" to represent a change in mobility, one could correct Equation (51):

No (Magnetic Field AND Surface) AND

No (Rotation AND Surface) $\rightarrow T$-Reversible $\rightarrow L$ Symmetr

And the corresponding modus tollens is:

(Magnetic Field OR Rotation) AND

Surface $\leftarrow$ Not $T$-Reversible $\leftarrow L$ Asymmetric

Combining Equations (53) and (59) leads to a contradiction:

Magnetic Field OR Rotation $\rightarrow$ (Magnetic Field OR Rotation) AND Surface (60)

Hence Equation (53) is incomplete and ceases to be applicable, allowing magnetic fields or rotation to coexist with the second law.

This can be illustrated as follows. In a uniform magnetic field and in the $a b$ sence of any surface, charged particles have untruncated circular orbits. Despite the curved orbits, an ensemble crossing any arbitrary volume has a symmetrical velocity distribution and zero mean velocity. The absence of surface removes any point of reference relative to which drift could occur. In other words, by symmetry of the ensemble, particles do not drift. The lack of information due to the statistical symmetry of particle motion makes energy extraction impossible, thereby upholding the second law.

Accordingly, Onsager's derivation of reciprocals could be adapted to make ensembles of microscopic fluctuations symmetrical even in the presence of magnetic fields and rotation. For example, his fluctuation analysis could be modified to apply to orbital parameters rather than plain linear variations, thereby removing the orbits' curvature as the source of the asymmetry.

In conclusion:

1) To be strictly correct, and to preserve the second law, Onsager should have excluded from reciprocals, those environments that include magnetic fields or rotation and a surface. However, this approach violates CPT symmetry because the surface enables parity.

2) In the absence of a surface, reciprocals do not violate the second law. They can be extended to magnetic and rotating environments by splitting the $L$ matrix into symmetric and antisymmetric parts. This is the approach taken by Mazur and de Groot [39]. 
3) Regardless of whether a surface is present, Onsager's exclusion violates CPT symmetry. Taking CPT seriously, his proof can be turned on its head, leaving one no choice but to conclude that when included with a mobility gradient such as a surface, magnetic fields and rotation can violate the second law.

\section{Appendix C. Expectation Value of $v_{x y}$ and $\nabla \mu \times B$}

The expectation value $\bar{v}_{\mu \times B}$ can be calculated from Equation (3) and from the given distribution which is $f_{M B}(v, \theta, \phi) \mathrm{d} v \mathrm{~d} \theta \mathrm{d} \phi$ for a maxwellian gas. When a particle is thermalized by a surface, the distribution is half Maxwellian, symmetrical around the normal on one side of the surface and zero on the other. Furthermore, since $v_{\mu \times B}$ in Equation (3) is only a function of $v_{x y}$ in the XY plane, the distribution can be further restricted to a $2 \mathrm{D}$ half Maxwellian distribution $f_{2 D M B / 2}\left(v_{x y}\right)$. Hence the expectation value for the drift velocity is:

$$
\bar{v}_{\mu \times B}=\int_{0}^{\pi} \int_{0}^{\infty} v_{\mu \times B} f_{2 D M B / 2}\left(v_{x y}\right) \mathrm{d} v_{x y} \mathrm{~d} \theta .
$$

Substituting Equation (3) into Equation (61) yields

$$
\bar{v}_{\mu \times B}=\int_{0}^{\pi} \int_{0}^{\infty}\left(v_{x y} \frac{\sin (\theta)}{\theta}\right) f_{2 D M B / 2}\left(v_{x y}\right) \mathrm{d} v_{x y} \mathrm{~d} \theta .
$$

Since the distribution is independent of $\theta$ in the range $0<\theta<\pi$ one can write:

$$
\bar{v}_{\mu \times B}=\int_{0}^{\pi} \frac{\sin (\theta)}{\theta} \mathrm{d} \theta \int_{0}^{\infty} v_{x y} f_{2 D M B / 2}\left(v_{x y}\right) \mathrm{d} v_{x y} .
$$

Defining the sine integral function as $\operatorname{Si}(\theta)=\int(\sin (\theta) / \theta) \mathrm{d} \theta$, and expressing the expectation value $\bar{v}_{x y}$ as:

$$
\bar{v}_{x y}=\int_{0}^{\infty} v_{x y} f_{2 D M B / 2}\left(v_{x y}\right) \mathrm{d} v_{x y} .
$$

yields:

$$
\bar{v}_{\mu \times B}=\operatorname{Si}(\pi) \bar{v}_{x y} .
$$

The expected velocity $\bar{v}_{x y}$ in Equation (65) can be calculated from the distributions $f_{M B}\left(v_{x}\right)$ and $f_{M B}\left(v_{y}\right)$. The full normalized distribution of $v_{x}$ for the range $-\infty<v_{x}<\infty$ is:

$$
f_{M B}\left(v_{x}\right)=\left(\frac{m^{*}}{2 \pi k_{B} T}\right)^{1 / 2} \exp \left(\frac{-m^{*} v_{x}^{2}}{2 k_{B} T}\right) .
$$

The presence of a surface restricts the range of $v_{y}$ to $0<v_{y}<\infty$. The distribution is half Maxwellian. Therefore, the normalization factor is twice as large for $v_{y}$ as for $v_{x}$. Therefore,

$$
f_{M B / 2}\left(v_{y}\right)=2\left(\frac{m^{*}}{2 \pi k_{B} T}\right)^{1 / 2} \exp \left(\frac{-m^{*} v_{y}^{2}}{2 k_{B} T}\right) .
$$

The 2D half-Maxwellian distribution for the velocity $v_{x y}$ in the XY plane is the joint probability of the two independent probabilities $f_{M B}\left(v_{x}\right)$ and $f_{M B / 2}\left(v_{y}\right)$ along each degree of freedom. Hence: 


$$
f_{2 D M B / 2}\left(v_{x}, v_{y}\right)=f_{2 D M B / 2}\left(v_{x}, v_{y}\right)=f_{M B}\left(v_{x}\right) f_{M B / 2}\left(v_{y}\right) .
$$

Therefore, substituting Equations (66) and (67) into (68) yields:

$$
f_{2 D M B / 2}\left(v_{x}, v_{y}\right) \mathrm{d} v_{x} \mathrm{~d} v_{y}=2\left(\frac{m^{*}}{2 \pi k_{B} T}\right) \exp \left(\frac{-m^{*}\left(v_{x}^{2}+v_{y}^{2}\right)}{2 k_{B} T}\right) \mathrm{d} v_{x} \mathrm{~d} v_{y} .
$$

Defining $v_{x y}^{2}=v_{x}^{2}+v_{y}^{2}$ and since $\mathrm{d} v_{x} \mathrm{~d} v_{y}=v_{x y} \mathrm{~d} \theta \mathrm{d} v$ :

$$
f_{2 D M B / 2}\left(v_{x y}, \theta\right) \mathrm{d} \theta \mathrm{d} v_{x y}=\left(\frac{m^{*}}{\pi k_{B} T}\right) \exp \left(\frac{-m^{*} v_{x y}^{2}}{2 k_{B} T}\right) v_{x y} \mathrm{~d} \theta \mathrm{d} v_{x y} .
$$

Equation (70) can be integrated to express $f_{2 D M B / 2}$ only in terms of $v_{x y}$. Integrating $\mathrm{d} \theta$ from $O$ to $\pi$ for the half maxwellian range yields,

$$
\begin{aligned}
f_{2 D M B / 2}\left(v_{x y}\right) \mathrm{d} v_{x y} & =\int_{0}^{\pi} f_{2 D M B / 2}\left(v_{x y}, \theta\right) \mathrm{d} \theta \mathrm{d} v_{x y} \\
& =\int_{0}^{\pi}\left(\frac{m^{*}}{\pi k_{B} T}\right) \exp \left(\frac{-m^{*} v_{x y}^{2}}{2 k_{B} T}\right) v_{x y} \mathrm{~d} \theta \mathrm{d} v_{x y} .
\end{aligned}
$$

Since

$$
\int_{0}^{\pi} \mathrm{d} \theta=\pi
$$

and substituting Equation (72) into Equation (71) produces:

$$
f_{2 D M B / 2}\left(v_{x y}\right) \mathrm{d} v_{x y}=v_{x y}\left(\frac{m^{*}}{k_{B} T}\right) \exp \left(\frac{-m^{*} v_{x y}^{2}}{2 k_{B} T}\right) \mathrm{d} v_{x y} .
$$

Therefore, the expected value $\bar{v}_{x y}$ is given by

$$
\bar{v}_{x y}=\int_{0}^{\infty} v_{x y} f_{2 D M B / 2}\left(v_{x y}\right) \mathrm{d} v_{x y}=\int_{0}^{\infty} v_{x y}^{2}\left(\frac{m^{*}}{k_{B} T}\right) \exp \left(\frac{-m^{*} v_{x y}^{2}}{2 k_{B} T}\right) \mathrm{d} v .
$$

Integrating,

$$
\bar{v}_{x y}=\sqrt{\frac{\pi k_{B} T}{2 m^{*}}} .
$$

Substituting into Equation (65) produces:

$$
\bar{v}_{\mu \times B}=\operatorname{Si}(\pi) \bar{v}_{x y}=\operatorname{Si}(\pi) \sqrt{\frac{\pi k_{B} T}{2 m^{*}}},
$$

and noting [40] that $\operatorname{Si}(\pi)=1.85$ and assuming effective mass $m^{*}=m$ then $\left(\pi k_{B} / 2 m^{*}\right)^{1 / 2}=48.79$ for electrons. Equation (76) yields $\bar{v}_{\mu \times B}= \pm 90.27 T^{1 / 2} \mathrm{~m} / \mathrm{sec}$.

\section{Appendix D. Entropically Driven $\nabla \mu \times B$ Drift}

Drift velocity $\bar{v}_{\mu \times B}$ corresponding to kinetic energy $\Delta U=\frac{1}{2} m^{*}\left(v_{\mu \times B}\right)^{2}$ is imparted to the particle along the $\mathrm{X}$ axis by the magnetic field. Using $\bar{v}_{\mu \times B}$ from Equation (18), one gets:

$$
\Delta U=\pi k_{B} T(\nabla \boldsymbol{\mu} \times \boldsymbol{B})^{2} .
$$

However, this energy is dissipated by the collision at the end of the mean free 
path (i.e., Boltzmann's Stosszahlansatz) which results in the production of entropy, and can be expressed as

$$
\Delta U=T \Delta S .
$$

Therefore, from Equation (77), the entropy generated by the dissipation of the drift energy is

$$
\Delta S=\pi k_{B}(\nabla \boldsymbol{\mu} \times \boldsymbol{B})^{2} .
$$

Since an entropic force generated by a change $\Delta S$ in entropy occurs along the entropy gradient and is given by [41]

$$
F=T \nabla(\Delta S) .
$$

Inserting the entropy gradient from Equation (79) into Equation (80) yields

$$
F=2 \pi k_{B} T \nabla \boldsymbol{\mu} \times \boldsymbol{B},
$$

which is a force along the $\overline{\boldsymbol{v}}_{\mu \times B}$ vector.

\section{Appendix E. $E \times B$ Drift Detailed Analysis}

This appendix follows the well-known Drude model [42]. A particle carried in an $E \times B$ drift follows a cycloid path described by the equation of motion

$$
\boldsymbol{F}=m\left(\frac{\mathrm{d}}{\mathrm{d} t}+\frac{1}{\tau}\right) \boldsymbol{v}=q(\boldsymbol{E}+\boldsymbol{v} \times \boldsymbol{B}),
$$

where $V$ is the particle's velocity, and $\tau$ is the average time between collisions with neutral atoms also assumed present in the chamber. This equation can be simplified by considering two ranges of applicability. First for very small values of $\tau$, collision frequency dominates, and we can write

$$
\frac{m \boldsymbol{v}}{\tau}=q(\boldsymbol{E}+\boldsymbol{v} \times \boldsymbol{B}) .
$$

Second, for very large values of $\tau$ for which cycloid behavior dominates, we can eliminate the differential term $\mathrm{d} / \mathrm{d} t$ by averaging the movement of the particles over one cycloid. This average is in fact the motion of the guiding centers. Rewriting the above equations in term of the velocity of the guiding center $\bar{v}$ averaged over one cycloid yields

$$
\frac{m \bar{v}}{\tau}=q(\boldsymbol{E}+\overline{\boldsymbol{v}} \times \boldsymbol{B}) .
$$

Particle motion expressed at the microlevel by Equations (84) can be represented at the macrolevel by substituting:

$$
\boldsymbol{J}=n q \overline{\boldsymbol{v}},
$$

yielding:

$$
\boldsymbol{J}=\frac{n q^{2} \tau}{m} \boldsymbol{E}+\frac{q \tau}{m} \boldsymbol{J} \times \boldsymbol{B} .
$$

If the particles have mobility $\mu$ and an effective mass $m^{*}$ in a given material, then the mean free time is: 


$$
\tau=\frac{\mu m^{*}}{q} .
$$

Applying Equation (87) into Equation (86) yields:

$$
\boldsymbol{J}=n q \mu \boldsymbol{E}+\mu \boldsymbol{J} \times \boldsymbol{B} .
$$

For simplicity, let the $\boldsymbol{E}$ vector span the XY plane and the $\boldsymbol{B}$ vector be in the $\mathrm{Z}$ direction. Rewriting Equation (88) in terms of $E_{x}, E_{y}$ and $B_{z}$ for the $J_{x}$ and $J_{y}$ component of $\boldsymbol{J}$ produces:

$$
J_{x}=n q \mu E_{x}+\mu B_{z} J_{y},
$$

and

$$
J_{y}=n q \mu E_{y}-\mu B_{z} J_{x} .
$$

Solving for $J_{x}$ and $J_{y}$ in terms of $E_{x} / B_{z}$ results in

$$
J_{x}=\frac{n q \mu B_{z}}{\left(1+\left(\mu B_{z}\right)^{2}\right)}\left(\frac{E_{x}}{B_{z}}+\mu E_{y}\right),
$$

and

$$
J_{y}=\frac{n q \mu B_{z}}{\left(1+\left(\mu B_{z}\right)^{2}\right)}\left(\frac{E_{y}}{B_{z}}-\mu E_{x}\right) .
$$

Equations (91) and (92) indicate that mobility can be used to vector the $E \times \boldsymbol{B}$ Drift. The angular direction $\theta_{E \times B}$ of the current vector $\boldsymbol{J}$ is

$$
\theta_{E \times B}=\tan ^{-1}\left(\frac{J_{y}}{J_{x}}\right)=\tan ^{-1}\left(\frac{E_{y}-\mu B_{z} E_{x}}{E_{x}+\mu B_{z} E_{y}}\right) .
$$

Defining $\alpha=E_{x} / E_{y}$, the equation above can be written as

$$
\theta_{E \times B}=\tan ^{-1}\left(\frac{1}{\mu B_{z}} \frac{1-\alpha \mu B_{z}}{1+\alpha / \mu B_{z}}\right) .
$$

The magnitude of the current $|\boldsymbol{J}|=\left(J_{x}^{2}+J_{y}^{2}\right)^{0.5}$ can be calculated from Equations (91) and (92) as:

$$
|\boldsymbol{J}|=\frac{n q \mu B_{z}}{\sqrt{1+\left(\mu B_{z}\right)^{2}}} \frac{\sqrt{E_{x}^{2}+E_{y}^{2}}}{B_{z}} .
$$

When $E_{x}=0$ the magnitude of the drift current is:

$$
|\boldsymbol{J}|=\frac{n q \mu B_{z}}{\sqrt{1+\left(\mu B_{z}\right)^{2}}} \frac{E_{y}}{B_{z}},
$$

and the direction of the drift is determined by the mobility of the particles:

$$
\theta_{E \times B}=\tan ^{-1}\left(\frac{1}{\mu B_{z}}\right) \text {. }
$$

Under an ideal, no-collision and infinite mobility, all particles drift in the same direction along the $\mathrm{X}$ axis, i.e., $\theta_{E \times B}=0, J_{x}=0$, and $J_{X}=n q E_{y} / B_{z}$. However, when mobility is finite, it can be negative for particles with negative charge (as it 
is defined in Equation (87)), which implies that particles with different charges drift in different directions.

Furthermore, as indicated by Equations (94) and (97), two factors contribute to redirecting or vectoring the drift away from the $\mathrm{X}$ direction. The first is $\alpha=$ $E_{x} / E_{y}$ which becomes significant when space charges accumulate downstream causing a back emf $E_{x} \neq 0$. The second is the product $\mu B_{z^{\prime}}$.

It is instructive to note that the $E \times B$ drift and the Hall Effect are two reciprocal perspectives of the same electromagnetic transport phenomenon. Both require a magnetic field. In the $E \times B$ drift, the input is an electric field and the output is a current. In the Hall Effect, the input is a current and the output is an electric field. 